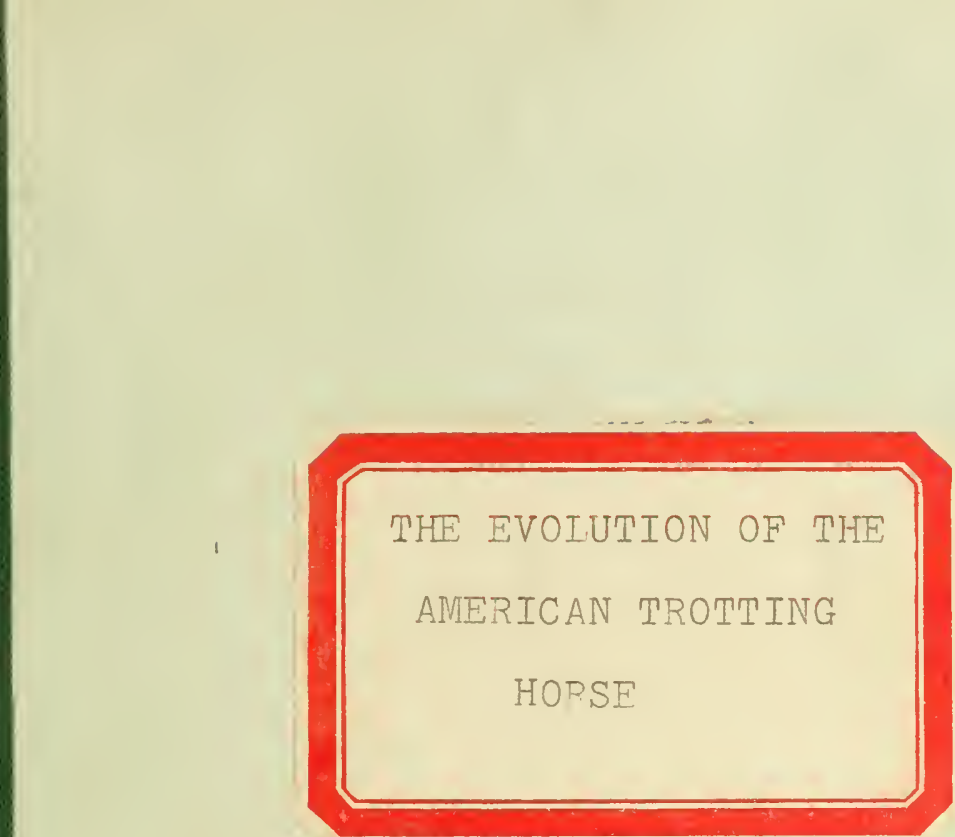




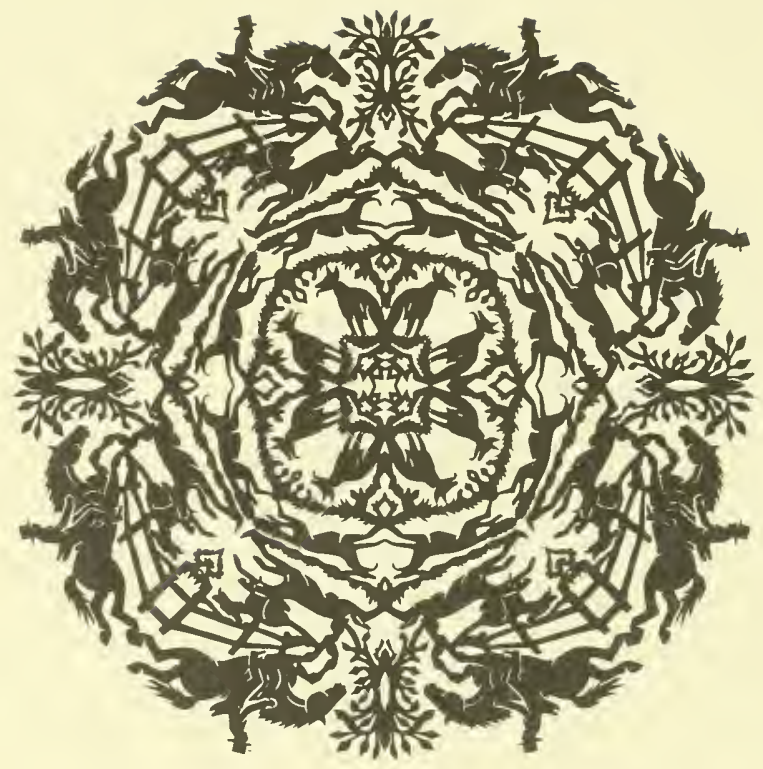

JOHN A.SEAVERNS 


\section{angany}

The, mean and extreme daily Temperatures in St. Louis for forty-seven years, as calculated from daily observations.

By DR. GEORGE ENGELMANN.

THE EVOLUTION OF THE AMERT'AN TROTTING-HORSE.

By FRANCIS E. Nं]'HER.

MAGNETIC SURVEY OF MISSOURI. 5th Annual Report.

By FRANCIS E. NIPHER.

ON THE EXPRESSION OF ELECTRICAL RESISTANCE IN TERMS OF A VELOCITY.

By FRANCIS E. NIPHER.

From the Trans. of the St. Louis Acad. of Science. Vol. IV., No. 3 .

Mareh, 1884. 

COMPLIMENTS OF

Frencis E. Nipleer. 
Digitized by the Internet Archive in 2009 with funding from Boston Library Consortium Member Libraries 


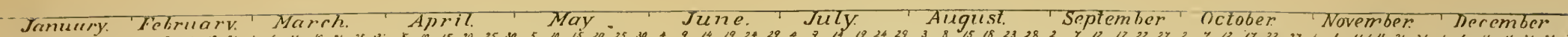

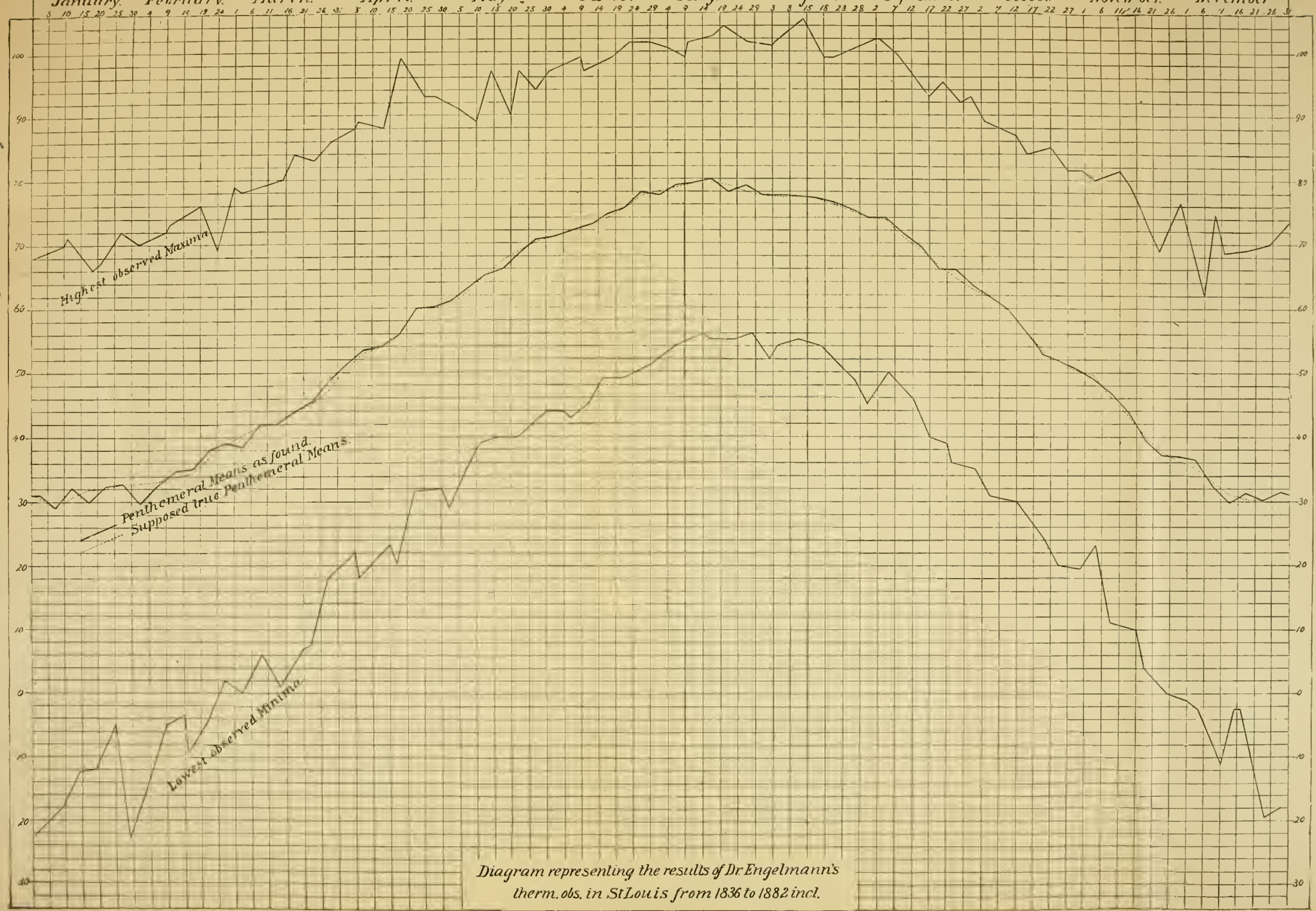



The mean and extreme daily Temperatures in st. Louis during forty stven years, as calculated from daily observations,

By Dr. George Engelmann.

Half a century has passed since I began to study the meteorology and climatology of this neighborhood, and since the year I 836 I have made regular meteorological observations, first on temperature, the winds and the condition of the sky, and soon afterwards on atmospheric pressure, rainfall and humidity.

I give here the results of my thermometrical observations, which I consider as the most important and most interesting of the series. They comprise, to be sure, only forty-seven years, and I might have waited until at least half a century was completed; but the results would scarcely have been different, and the task then perhaps problematical of accomplishment.

The observations were made within the city of St. Louis, and can thus not claim precision for this whole region. St. Louis, to be sure, was, when they commenced, a small town of perhaps I 5,000 inhabitants, while now, at their completion, it is a large city of probably 400,000 , with the necessary accompaniment of brick and stone, and especially with the smoke of thousands of chimneys, furnaces and factories, and the almost total absence of verdure. It has been held by some, that these influences had little effect on temperature, but that brisk breezes would soon dispel smoke and equalize temperature. This, however, is not quite so, and direct thermometrical comparisons prove that the extreme temperatures, and, remarkably enough, even the extreme heat, are less marked in the city than in the country, and that the mean temperature is higher in the city than in the country (Trans., vol. ii., p. 70); but, aside from instrumental observation, the state of the vegetation proves it every spring and fall, when we find in our city gardens the plants uninjured on mornings when in the country they have suffered from late or early frosts.

St. Louis lies very nearly in the centre of the Mississippi Valley, 600 miles north of the Gulf of Mexico and just as far south of Lake Superior, about 500 miles west of the Alleghanies and Soo miles east of the Rocky Mountains; its Washington University, one 
mile west of the river, lies in Lat. $3^{\circ} 3^{8^{\prime}} \mathrm{O} 3^{\prime \prime}$ and Long. $90^{\circ} \mathrm{I}^{\prime}$ ${ }^{1} 5^{\prime \prime}$; the low-water mark of the Mississippi is 379 feet above the Gulf, and the foot of Market street (City Directrix) is $4 \mathrm{r} 3$ feet above the same.

My observations were made in the first twelve years on the south-east corner of Second and Chestnut streets, only two blocks from the river and 75 feet above low-water mark of 1863 ; for the next 22 years on the south-west corner of Fifth and Elm streets, five blocks from the river and 110 feet above low-water mark; and for the last 13 years on the north-west corner of Thirtieth and Locust streets, two miles from the river and 177 feet above lowwater mark. When 1 was absent from the city Dr. A. Wislizenus and lately Mr. B. D. Kribben have kindly filled the gaps.

My instruments were at first such as could then be obtained here; soon I imported correct thermometers from Europe, and for nearly 40 years I used those made by Jas. Green, then of Baltimore, and soon afterwards of New York.

For many years the observations were made at different periods of the day, and especially at hours when the cxtremes might be expected to occur, viz. at sunrise and at 3 P.M.; and the maximums and minimums were selected from all of them, often eight in a day, at whatever hour of the day they were found. Differential thermometers were observed only since the last 12 years. Thus I may not always have noted the absolute extremes of each day, and my tables can claim only approximate reliability; I give them for what they may be worth, but I can assure my readers that they furnish a record elaborated with zeal, conscientiousness, care and assiduity, and for a length of time such as probably few others, if any, exist in this valley.

The arrangement of the tables explains itself. The first two columns represent the means of the 47 minimum and of the 47 maximum observations made on each day of the year, and the third column the mean calculated from the two former. The next "Min." column gives the lowest and the "Max." column the highest temperature ever observed on that day; the column of years next to these gives the year in which these extremes did occur. The last column represents a supposed-or estimated-true mean for the day after eliminating excessive extremes.

The year has been divided into 73 periods of 5 days each, the 
means of which are printed in full-face type, to distinguish them. It will be seen, however, that the means of these periods do not progress, rise and fall, much more evenly than the single daily means ; compare, e.g., the mean of Jan. $3^{\text {Ist }}$ to Feb. $4^{\text {th, which }}$ is so much lower than the mean of the foregoing or the following five days that one might suspect a regular and normal decline of temperature in these days, and not a mere accident.

A few facts must strike every one who examines the tables. The first is, that a time even as long as 47 years fails to give us anything approaching absolute and reliable means; and we come to the painful conclusion, that observations even continued for double that time, or for a century, may not yet obtain that desirable object. It seems that the excessive extremes of one or of a few days such as we often observe in our climate of extremes, especially in the winter season, will influence-or, I may say, vitiate-the means of a long series of observations; and the question with me arises, whether such extremes ought not to be eliminated from the series, and thus truer means be obtained. At the same time we may justly be astonished that from such heterogeneous data so much order and system result-which gives us hope that we cannot be quite on the wrong track.

Another fact, which strikes us in looking over the tables, is that the mean temperatures do not increase and decrease evenly, but sometimes quite rapidly, and at other times they may become almost stationary for a time. These points come out most strikingly on a diagram which embodies the essential parts of the results and shows the daily progress of the temperature. Thus we find very little change from the middle of December to the first part of February, though the temperature proves to be lowest from January $4^{\text {th }}$ to $13^{\text {th }}$; then we notice a rapid rise from Feb. 6th to 20 th, a slower rise to the middle of March, then a rapid one to the end of the month ; in the forepart of April a slow and after the middle of that month a very marked one; then follows a tolerably even, at last quite slow, rise to July 9 th, when between this date and the I 3 th the greatest elevation of the curve is obtained. After that the mean temperature falls slowly to the middle of August, followed by a more rapid decline to the end of September; after a slight pause in the first week in October, a more rapid fall takes place for the following two weeks and a 
slighter one in the two weeks succeeding them. After that the temperature sinks rapidly to about Dec. Ioth, from which time till the beginning of February the changes are not very marked.

The mean temperature of April I 7 th to I 9 th and from October I 2 th to $7^{\text {th }}$ correspond with the mean of the year.

The tables, and still more distinctly the diagram, show us also that the extreme highest and lowest temperatures diverge most in winter and least in summer, and that their values are much more variable in the former than in the latter season. The possibilities of range from the middle of December to the middle of March are So to 95 degrees, while in June and July they amount only to 40 or 45 degrees.

The same law is found when we compare the actually observed lowest minimum and highest maximuni of every month; their divergence is greatest in January, and least, not much more than half, in July.

\begin{tabular}{|c|c|c|c|c|c|c|c|}
\hline Jan & $\begin{array}{r}\text { Min, } \\
-22.5\end{array}$ & $\begin{array}{l}\text { Max. } \\
72.0\end{array}$ & $\begin{array}{r}\text { Range. } \\
94.5\end{array}$ & July & $\begin{array}{l}\text { Min. } \\
53.0\end{array}$ & $\begin{array}{l}11 \mathrm{ax} . \\
104.0\end{array}$ & $\begin{array}{r}\text { Range. } \\
51.0\end{array}$ \\
\hline February & -15.0 & 76.0 & 91.0 & August & 45.0 & 104.0 & 59.0 \\
\hline March ... & 0.0 & 86.0 & 86.0 & September... & 35.0 & 102.0 & 67.0 \\
\hline April ......... & 18.0 & 99.0 & 81.0 & Oetober ....... & 19.5 & 910 & 71.5 \\
\hline IIay...$\ldots \ldots$ & 29.0 & 975 & 68.5 & November.... & -0.5 & 81.5 & 82 . \\
\hline June & 43.0 & 101.5 & $58 . \overline{5}$ & December.... & -19.5 & 72.5 & 0 \\
\hline
\end{tabular}

Nearly the reverse is the case-i.e. the range in winter is much smaller than that in summer-if we compare the difference of the average daily minima and maxima for each month:

January .. $13.27 \mid$ April.... 18.29| July ... 18.2t October .. 18.00 February . 14.72 May..... 18.77 August... 17.75 November 14.06 March... 16.40 June .... 18.14 Septenber 19.05 December. 11.97

The range, it will be seen, is, on the whole, least in the cooler and greatest in the warmer months of the year; but this difference is not due to the lower or higher temperatures of those months, for it will be seen that in December the range is the smallest (smaller than in January) and in September greatest (greater than in July). This variation in the range of maxima and minima is undoubtedly owing to the condition of the sky in the different months. Gloomy weather prevails in the beginning of winter, and a clear sky with abundant evaporation, and thus a reduction of night temperature, in the autumn. The little table can give us an indication of the prevailing weather in the different months. Thus the difference, and its cause, the clearness of 
the sky, rises from December gradually till May, falls a little in June and July and more in August, rises to its highest point in September, is yet high in October and then falls rapidly till December, to rise again in January.

The temperature of our continental locality shows a great difference from that of the western coast of Europe ; as a convenient example we may refer to the temperature of London. Their winters are warmer from the latter part of November until the beginning of March, and their summers much cooler from this period to the latter third of November; and the mean is much higher here. Whole Jan. Feb. Mar. Apr. May. June. July. Aug. Sept. Oct. Nov. 1ec. Year. $\begin{array}{llllllllllllll}\text { St. Louis.. } & 31.8 & 35.4 & 43.7 & 56.2 & 66.3 & 74.7 & 79.2 & 76.8 & 69.0 & 56.1 & 42.8 & 33.4 & 55.4\end{array}$

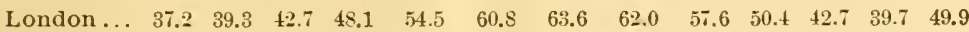
Difference. $+5.4+3.9-1.0-8.1-11.8-13.9-15.6-14.8-11.4-5.7-0.1+6.3-5.5$

MEAN AND EXTREME TEMPERATURES IN ST. LOUIS FROM 1836 to 1882.

\begin{tabular}{|c|c|c|c|c|c|c|c|c|}
\hline \multirow[t]{2}{*}{1836.1882} & \multicolumn{3}{|c|}{$\begin{array}{l}\text { Mean Values for each } \\
\text { Day. }\end{array}$} & \multicolumn{4}{|c|}{$\begin{array}{l}\text { Extreme Maxima and Minima observed } \\
\text { on each Day of the Year. }\end{array}$} & \multirow{2}{*}{$\begin{array}{l}\text { Sup- } \\
\text { posed } \\
\text { true } \\
\text { Mean. }\end{array}$} \\
\hline & Min. & Max. & Mean. & Min. & Year. & Max. & Year. & \\
\hline \multirow[t]{30}{*}{ Jan. } & 23.55 & 36.77 & 30. & -22.5 & 1864 & 68.0 & $\overline{18}$ & 31.5 \\
\hline & 25.35 & 37.97 & 31. & -13.0 & 1879 & & 1855 & 31.3 \\
\hline & 25.54 & 38.55 & 32.04 & -15.5 & 16 & 66.5 & $187 \mathrm{t}, 1880$ & 31.0 \\
\hline & 23.95 & 35.82 & 29.88 & -6.0 & "6 & 63.5 & 1880 & 30.5 \\
\hline & 23.45 & 39.22 & 31.33 & -8.5 & 1864 & 62.0 & 1876 & 30.2 \\
\hline & 24.36 & 37.66 & 31.01 & -13.1 & & $6 . \overline{1} 1$ & & 30.9 \\
\hline & 23.76 & 37.63 & 30.69 & -9.0 & 1879 & 64.0 & 1880 & 30.0 \\
\hline & 22.33 & 33.95 & 28. & -1.0 & 18 & 64 & 18 & 29.4 \\
\hline & 22.75 & 34.61 & 28 . & -6.0 & 18 & 68 & 6 & 29.0 \\
\hline & 22.65 & 35. & 29 & -18.0 & & & & 29.0 \\
\hline & 22.67 & 34.82 & 28.74 & -11.0 & 1881 & 70.0 & 1839 & 29.5 \\
\hline & 20.83 & 3.9 .29 & 29.06 & -9.0 & & 66.3 & & $\mathbf{2 9 . 4}$ \\
\hline & 25.42 & 39.20 & 32.31 & -1.0 & 18 & 7 & 39 & 30.0 \\
\hline & 26.69 & 39.27 & 32.5 & -2.0 & 18 & 60 & 1863 & 30.2 \\
\hline & 24.61 & 36.81 & 30 . & 0.0 & & 59 & & 30.6 \\
\hline & 5.61 & $3 \varsigma .11$ & 31 & -12.5 & 18 & 6 & 8 & 31.0 \\
\hline & 26.71 & 39.67 & 33.19 & -2.5 & 187 & 66.0 & 1847 & 31.4 \\
\hline & 2.5 .50 & 38.61 & 32.21 & -60 & & 6.5 .0 & & 30.6 \\
\hline & 24.03 & 36.27 & 30.15 & 2.0 & 184 & 63.0 & 1845 & 31.8 \\
\hline & 21.09 & 34.27 & & -11.0 & & & 18 & 32.0 \\
\hline & 21.65 & .24 .32 & 27. & -12.5 & 18 & 66.0 & " & 32.0 \\
\hline & 23.63 & 38.09 & 30.8 & -12.0 & 18 & 61.0 & 1843 & 32,0 \\
\hline & 27.83 & 39.54 & 33.68 & -1.5 & 1866 & 64.0 & 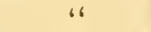 & 32.0 \\
\hline & 23.64 & $3(6.49$ & 30.07 & -7.0 & & (5:3.6) & & 31.9 \\
\hline & 25.97 & 35.79 & 32.38 & -3.0 & 185 & 67.0 & 1843 & 32.0 \\
\hline & 23.84 & 39.1 & 31. & -2.5 & 18 & 62 & 18 & 32.0 \\
\hline & 24.63 & 38.36 & 31.4 & 0.0 & 18 & 62.0 & 18 & 32.0 \\
\hline & 25.57 & 39.64 & 32.60 & 2.0 & 18 & 65.0 & 18 & 32.2 \\
\hline & 27.26 & 41.63 & 34.44 & -55 & 1840 & 65.0 & 1864 & 32.4 \\
\hline & 25.4 & 39.5 & 32.45 & -1.8 & & 64.2 & & 32.1 \\
\hline
\end{tabular}


5OI ENGELMANN-MEAN \& EXTREME TEMPERAT'E IN ST. LOUIS.

\begin{tabular}{|c|c|c|c|c|c|c|c|c|}
\hline \multirow[t]{2}{*}{$1836 \cdot 1882$} & \multicolumn{3}{|c|}{$\begin{array}{c}\text { Mean Value for each } \\
\text { Dav. }\end{array}$} & \multicolumn{4}{|c|}{$\begin{array}{l}\text { Extreme Maxima and Minima observed } \\
\text { on each Day of the Year. }\end{array}$} & \multirow{2}{*}{$\begin{array}{l}\text { Sup. } \\
\text { posed } \\
\text { true } \\
\text { Mean }\end{array}$} \\
\hline & Min. & Max. & Mean. & Min. & Year. & Max. & Year. & \\
\hline \multirow{2}{*}{$\begin{array}{r}\text { Jan. } 26 \\
27\end{array}$} & 26.76 & 40.36 & 33. & 1.0 & 1865 & 71.5 & 1843 & 32.6 \\
\hline & 26. & & & -0.5 & & & & 32.6 \\
\hline 28 & 24.85 & 39. & & -6.5 & & 67.0 & & 32.7 \\
\hline \multirow{3}{*}{$\begin{array}{l}29 \\
30\end{array}$} & 25.32 & 3930 & & -23.0 & "6 & 64.5 & 1852 & 32.8 \\
\hline & 24.79 & 41.09 & 3 & -2.0 & 1856 & 64.5 & 1842 & 32.8 \\
\hline & 25.73 & 39.88 & 32 & -6.2 & & 67.8 & & 32.7 \\
\hline 31 & 24.96 & 38.20 & & 2.0 & 1875 & 65.5 & 1877 & 32.8 \\
\hline Feb. 1 & 23.32 & 37.20 & & -8.5 & 1836 & 70.0 & & 32.8 \\
\hline & 23.55 & 35.77 & & -3.5 & 1873 & 56 & 1846 & 32.9 \\
\hline \multirow{2}{*}{$\begin{array}{l}3 \\
4\end{array}$} & 21.71 & 35. & & -15.0 & 1856 & 62.5 & 1852 & 32.9 \\
\hline & 22.01 & 36.51 & 29.26 & -11.0 & "6 & 61.0 & 1837 & 33.0 \\
\hline \multirow[b]{2}{*}{5} & 23.11 & 36.68 & 29.89 & -7.2 & & 63.0 & & 32.9 \\
\hline & 24.67 & 39.29 & & -3.5 & 1856 & 6 & 1837 & 33.0 \\
\hline 6 & 26 . & 41 & & 3.0 & 18 & & & 33.1 \\
\hline & 26.08 & 41. & & -3.5 & 1872,1875 & 65 & & 33.1 \\
\hline & 24.74 & 39. & & 2.0 & 1842 & 66 & 1847 & 33.2 \\
\hline & 24.37 & 40.05 & 32.21 & -5.0 & 1875 & 72.0 & 1876 & 33.3 \\
\hline & 25.17 & 40.31 & 32.47 & -1.4 & & 66.2 & & 33.1 \\
\hline 10 & 25.92 & 41.56 & 33.74 & 1.0 & 1841 & & 1876 & 33.5 \\
\hline 11 & & & & & & & & 33.7 \\
\hline & 29.00 & 43 & 36 & 2.0 & 6 & 7 & & 33.9 \\
\hline & 29.06 & 40 & & 4 & 18 & & & 34.0 \\
\hline 14 & 24.71 & 39.38 & 32.04 & -3.5 & 186 & 64.0 & 1857 & 34.1 \\
\hline & & 41.74 & 34.73 & 1.2 & & 69.9 & & 33.8 \\
\hline 15 & 26.53 & 41.09 & 33 & -9.5 & 1866 & & 848 & 34. \\
\hline 16 & & & & -5.0 & & & 7 & 34. \\
\hline 17. & 28. & 41. & 34 & -4.0 & & & & 34 \\
\hline 18 & 28.05 & 42.3 & 35 & -2.5 & & 65 & 1873 & 34. \\
\hline 19 & 30.26 & 44.57 & 37.41 & 5.0 & 1838 & 76.0 & 1859 & 35. \\
\hline & 97.7 & 42.36 & 335.07 & -3.2 & & 69. & & 34.0 \\
\hline 20 & 31.83 & 45.80 & & -3.5 & 18 & & 18 & 35. \\
\hline 21 & 30. & & & -4 & & & & 36. \\
\hline 22 & & & & 1. & 18 & & & 36. \\
\hline 23 & 30.1 & & & 0 & & & & 37. \\
\hline 24 & 29.38 & 45.91 & 37 & 6 & 1873 & 69. & 1880 & 37. \\
\hline & & 45. & 38.21 & -0.1 & & & & 36. \\
\hline 25 & 31 & 46 & & 7. & & & 187 & 38. \\
\hline 26 & 31.68 & & & & & & & 38 \\
\hline 27 & & & & & & & & 38 \\
\hline 28 & 30. & & & 10.5 & 1836,1869 & 74 & 1861 & 39. \\
\hline 1 & 32.35 & 49.02 & 40 & 10.0 & 1843 & 79 & & 39. \\
\hline & |:31.22 & 47.29 & & & & 72 & & 38. \\
\hline 2 & 31.36 & 45 & & 8 & & 76 & 18 & 39 \\
\hline 3 & \begin{tabular}{l|l|}
3 & 29.29
\end{tabular} & 43. & & 0. & & & 18 & 40 \\
\hline & +29.50 & 44.54 & & 6 & & 75 & 18 & 40 \\
\hline & 30 & & & 8. & & 71 & 18 & 40 \\
\hline & \begin{tabular}{l|l}
34 \\
34
\end{tabular} & 49.20 & 42.07 & 4.5 & 1869 & 76.5 & 1860 & 41 \\
\hline & & & & & & 75.4 & & 40. \\
\hline & $\begin{array}{l}7 \quad 34 \\
-15\end{array}$ & & & 10. & 18 & 77.0 & 1853 & 41 \\
\hline & & & & 13. & & 77.0 & 18 & 41 \\
\hline & 35.01 & 48.18 & & & & 78.5 & 18 & 41 \\
\hline 10 & \begin{tabular}{l|l}
03.22 \\
\end{tabular} & & & 10.0 & & & 12 & \\
\hline 11 & & 49. & 42.11 & 7. & 1836 & 69.0 & 1848 & 42. \\
\hline & $34.4: 3$ & 49.85 & $4 \because .14$ & 7.4 & & 76.0 & & 11 \\
\hline
\end{tabular}




\begin{tabular}{|c|c|c|c|c|c|c|c|c|}
\hline \multirow[t]{2}{*}{$1836-1882$} & \multicolumn{3}{|c|}{$\begin{array}{l}\text { Nean Value for each } \\
\text { Day. }\end{array}$} & \multicolumn{4}{|c|}{$\begin{array}{l}\text { Extreme Maxina and Minima observed } \\
\text { for each Day of the Year. }\end{array}$} & \multirow{2}{*}{$\begin{array}{l}\text { Sup- } \\
\text { posed } \\
\text { true } \\
\text { Mean } \\
\end{array}$} \\
\hline & Min. & Max. & Mean. & Min. & Year. & Max. & Year. & \\
\hline $\operatorname{Iar} 12$ & 34.82 & 50.86 & 42.84 & 11.5 & 1836,1857 & 71.0 & 1839,1861 & 42. \\
\hline 13 & 34.24 & 50.64 & 42.44 & 5.0 & 1867 & 75.0 & 1850 & \\
\hline 14 & 34.51 & 50.55 & 42.53 & 1.0 & & 76.5 & & 42. \\
\hline 15 & 33.87 & 48.89 & 41.38 & 7.5 & 1870 & 80.5 & 1854 & 42. \\
\hline 16 & 34.14 & 50.03 & 42.08 & 9.0 & 1843 & 77.5 & 1868 & 42. \\
\hline & 34.31 & 50.19 & 42.25 & 6.8 & & 76.1 & & 42. \\
\hline 17 & 33.49 & 52.07 & 42.78 & 10.5 & 1879 & 79.0 & 1842 & 43. \\
\hline 18 & 34.86 & 52.32 & 43.59 & & & & & 43. \\
\hline 19 & 36.09 & 54.82 & 45.45 & 18.5 & 187 & 84 & " & 44. \\
\hline 20 & 36.91 & 52.52 & 44.71 & 14 & & & " & 44. \\
\hline 21 & 34.21 & 50.68 & 42.44 & 7.0 & 1876 & 76.0 & 1878 & 44. \\
\hline & 35.11 & 52.48 & 43.79 & 13.0 & & 81.4 & & 43. \\
\hline 22 & 35.56 & 54.04 & 44.80 & 13.0 & 1843 & 76.0 & 1857 & 45. \\
\hline 23 & 37.09 & 55.48 & 46.28 & 7.5 & & & & 5. \\
\hline 24 & 36.90 & 54.30 & 45.60 & 12.0 & " & 83 & & 45 \\
\hline 25 & 37.21 & 53.76 & 45.48 & 13 & " & 82 & 1802 & 45. \\
\hline 26 & 37.69 & 54.92 & 46.30 & 13.5 & 1873 & 78.5 & 1838 & \\
\hline & 36.89 & 54.50 & 45.69 & 11.8 & & 80.5 & & \\
\hline 27 & 39.16 & 56.81 & 47.98 & 23.5 & 185 & 85.0 & $18:$ & 6. \\
\hline 28 & 39.77 & 57. & 48.54 & 18 & & 83 & & . \\
\hline 29 & 40.42 & 58.31 & 49.36 & 23. & 1876 & 86 & 2 & . \\
\hline 30 & 41.07 & 57. & 49. & 28.0 & ' & 84 & 1838 & \\
\hline 31 & 40.35 & 57.34 & 48.84 & 25.5 & 1856 & 84.0 & “" & \\
\hline & 40.1.5 & 57.53 & 48.84 & 23.7 & & 84.5 & & 47. \\
\hline pr. 1 & 39.70 & 57.25 & 48.47 & 24. & 1881 & & 1882 & 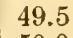 \\
\hline & $40.9 \pm 3$ & 61.12 & 51.02 & 24.0 & ' & 85 & & 0. \\
\hline 3 & 44.66 & 62.44 & 53.55 & 24. & 187 & & " & 01 \\
\hline 4 & 44.00 & 59.67 & 51. & 23 & & & $"$ & 5 \\
\hline 5 & 42.79 & 60.82 & 51.80 & 22.5 & 1857 & 88.5 & 1871 & 2 \\
\hline & 42.41 & 60.26 & 51.33 & 23.6 & & & & 0. \\
\hline 6 & 43.61 & 62.89 & 53.25 & 18 & 18 & S? & 18 & 52 \\
\hline 7 & 44.77 & $6:$ & 54. & & & & & 3 \\
\hline 8 & 45.59 & 60.96 & 53. & 24 & 18 & $8:$ & 18 & 53 \\
\hline 9 & 43.84 & 61 & 52. & 27.0 & 185 & & & 53 \\
\hline 10 & 45.07 & 61.94 & 53.50 & 28.5 & 1836,1874 & 87 & & 53 \\
\hline & 44.57 & 62.33 & 53.45 & 25.3 & & $8:$ & & 53. \\
\hline 11 & 43.81 & 62.53 & 53.17 & 27. & 185 & 83 & & 53 \\
\hline 12 & 45. & & 53. & & & & & 53 \\
\hline 13 & 45 & & & & " & & & \\
\hline 14 & 45.03 & 63.26 & 54.14 & 28.5 & 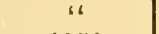 & 84 & & U \\
\hline 15 & 44.52 & 62.02 & 53.27 & 23.0 & 1850 & 82.0 & 1856 & 54 \\
\hline & & & & & & & & \\
\hline 16 & 45.62 & 62 & 53. & & 1875 & & 18 & \\
\hline 17 & 45. & & 54 & 20 & 4 & & & \\
\hline 18 & 45.78 & 64.34 & 55. & 26 & " & 99.0 & & 56 \\
\hline 19 & 47.20 & & & 29.0 & 1857 & & 18 & 57 \\
\hline 20 & 48.47 & 67.92 & $5 \$ .19$ & 34.0 & " & 85.0 & 1836 & 80 \\
\hline & 46. & & 5.5 & 27.2 & & 90.2 & & -0 \\
\hline 21 & 4976 & 69.01 & & 36.0 & 1857,1875 & 85.0 & 18 & \\
\hline 22 & 51.37 & & & & & 87.5 & 18 & 0 \\
\hline 23 & 49.88 & 69. & 59. & 31.5 & 186 & 87.0 & 1842,1854 & 60. \\
\hline 24 & 50.24 & 68.92 & 59. & 34.0 & 1874 & 88.5 & 1854 & 60. \\
\hline 25 & 51.89 & 70.82 & 61.35 & 33.5 & 1874,1875 & 93.0 & 1813,1855 & 60. \\
\hline & 50.6 & 69.67 & $60.1)$ & 39.8 & & 85.2 & & 9 \\
\hline
\end{tabular}


503 ENGELMANN-MEAN \& EXTREME TEMPERAT'E IN ST. LOUIS.

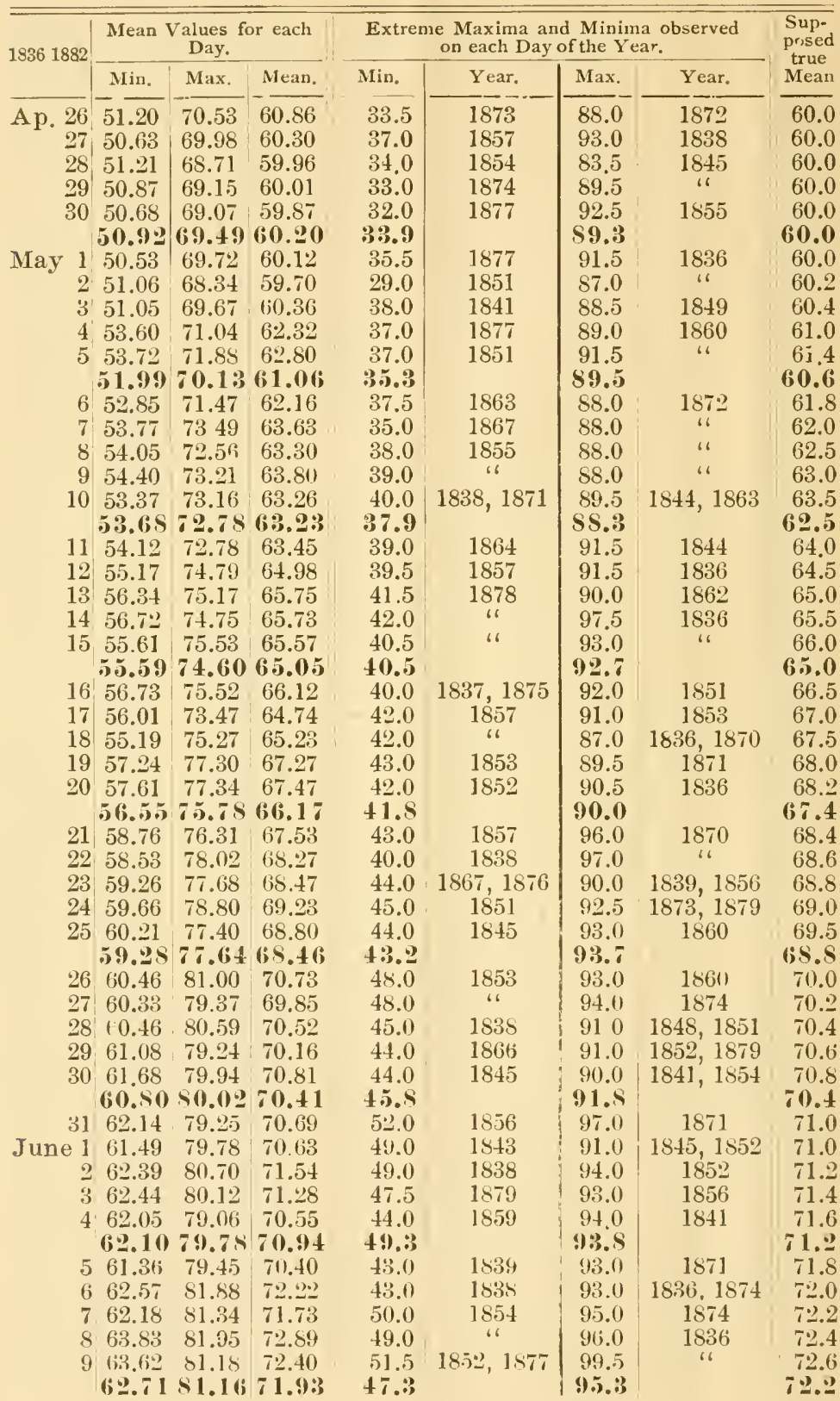




\begin{tabular}{|c|c|c|c|c|c|c|c|c|}
\hline \multirow[t]{2}{*}{$1836 \cdot 1882$} & \multicolumn{3}{|c|}{$\begin{array}{c}\text { Mean Values for each } \\
\text { Day. }\end{array}$} & \multicolumn{4}{|c|}{$\begin{array}{c}\text { Extreme Maxima and Minima observed } \\
\text { on each Day of the Year. }\end{array}$} & \multirow{2}{*}{$\begin{array}{l}\text { Sup- } \\
\text { posed } \\
\text { true } \\
\text { Mean } \\
\end{array}$} \\
\hline & Min. & Max. & Mean. & Min. & Year. & Max. & Year. & \\
\hline 1n. 10 & 62.65 & 81.90 & 72.27 & 46.5 & 1877 & 97.5 & 1836 & 72.8 \\
\hline 11 & 61.90 & 79 & 70 & & & & & 73.0 \\
\hline 12 & 63.32 & 81.59 & & 50 & 18 & 9 & & 73.2 \\
\hline 13 & 64.87 & 83.82 & 74 & 50 & & 94.5 & & 73.4 \\
\hline \multirow[t]{2}{*}{14} & 65.01 & 83.84 & 74.42 & 51.0 & 1856 & 96.0 & 1879 & 73.6 \\
\hline & 63.55 & $\$ 2.10$ & 72.82 & 48.5 & & 95.3 & & 73.2 \\
\hline 15 & 65.11 & 83.79 & 74.45 & $49.0^{\circ}$ & 1869 & 96.5 & 1868 & 73.8 \\
\hline 16 & 65.26 & 82.32 & 73.79 & 53.0 & 1841 & 98.5 & & 74.0 \\
\hline 17 & 65.56 & $\$ 2.60$ & 74 & 5 & 1876 & & "6 & 74.3 \\
\hline 18 & 65.78 & 83.97 & & 49 & & 99.0 & ." & 74.6 \\
\hline \multirow[t]{2}{*}{19} & 65.59 & 84.37 & 74. & 52.0 & 1866 & 96.0 & 1853,1869 & 74.8 \\
\hline & 65.46 & $5: 3.41$ & 74.43 & 51.0 & & 97.7 & & 74.3 \\
\hline 20 & 65.92 & 83.96 & 74.94 & 52 & 1862 & 98.0 & 1861 & 75.0 \\
\hline 21 & 65.57 & 83.52 & & & & & 18 & 5.3 \\
\hline 22 & 65.64 & 85.82 & & & 18 & 9 & 1871 & 75.6 \\
\hline 23 & 67.06 & 84.58 & & & " & 101.5 & & 76.0 \\
\hline \multirow[t]{2}{*}{24} & 66.91 & 85.11 & 76. & 55.5 & 1853 & 97.5 & 1870 & 76.5 \\
\hline & 66.22 & $\$ 4.60$ & 75.41 & 51.9 & & & & 5.7 \\
\hline 25 & 68.13 & 86.54 & 77 & 5 & 1852 & 98.0 & 1870,188 & 77.0 \\
\hline 26 & 68.73 & 87.88 & & & & 98.5 & & 77.5 \\
\hline 27 & 70.26 & 87.21 & & & & & & 8.0 \\
\hline 28 & \begin{tabular}{|l|l}
69.36 \\
\end{tabular} & 87.48 & & 5 & 1866 & 10 & 1870 & 78.0 \\
\hline \multirow[t]{2}{*}{29} & 69.26 & 86.08 & $7 \pi$. & 51.0 & & 101.5 & & 88.0 \\
\hline & 69.15 & 87.04 & 78.09 & 5.5 .3 & & 99.0 & & 7.7 \\
\hline 30 & 69.23 & $\$ 6.95$ & 78 & 56 & 1871 & 101.5 & 1870 & 8.0 \\
\hline \multirow[t]{11}{*}{ July 1} & 68.39 & 85.91 & & 54 & 1851 & & & 78.0 \\
\hline & 68.06 & 85.60 & & & & & & 78.0 \\
\hline & 68.42 & 87 & & & & & & 78.1 \\
\hline & 68.71 & 87.89 & 78. & 53.0 & 1859 & 100.5 & 1868 & 78.2 \\
\hline & 68.56 & 56.69 & 77. & .54 .6 & & 99.6 & & 78.1 \\
\hline & 69.24 & 87.39 & 78. & & 1882 & 98.5 & & 78.3 \\
\hline & 70.11 & & & & & & & 8.6 \\
\hline & 70.20 & 87.84 & & & & 98.0 & 18 & 79.0 \\
\hline & 70.55 & 87.97 & & & 1870 & 97.0 & $1 \varepsilon 54,1879$ & 79.0 \\
\hline & 71.05 & 89.15 & & 57 & $18+2$ & 99.0 & 1858 & 79.2 \\
\hline & 70.23 & 55.05 & 79 & 5 & & 97.9 & & 78.5 \\
\hline 10 & 71.46 & 87. & & & 10 & & & 79.2 \\
\hline 11 & & & & & 1854,1873 & & & 9.4 \\
\hline 12 & 70.64 & & & & & & & 9.4 \\
\hline 13 & 69.99 & 88 & & & & & 18 & 79.6 \\
\hline \multirow[t]{2}{*}{14} & 70.69 & 88.66 & 79. & 56.5 & 1882 & 100.5 & 1868 & 79.6 \\
\hline & & & & & & & & 9.4 \\
\hline 15 & 71.44 & $\$ 9.38$ & & & 2 & & & 80.0 \\
\hline 16 & 71.42 & $\$ 9.02$ & 80 & & & & & 80.0 \\
\hline 17 & 70.66 & & & & & & & 80.0 \\
\hline 18 & 70.77 & & & & & & & 79.6 \\
\hline \multirow[t]{2}{*}{19} & 70.44 & 87.70 & 79.6 & 58.0 & 18 & 100.0 & 185 & 79.4 \\
\hline & & +55.95 & & & & 100,8 & & 79.8 \\
\hline 20 & 69.94 & 86.92 & & & 18 & 100.5 & 185 & 79.2 \\
\hline & 69.08 & & & & & & & 79.0 \\
\hline 22 & 68.63 & 85.63 & 77. & 57.0 & 1864 & 101.5 & 1870 & 79.0 \\
\hline 23 & 68.72 & 87.16 & & 55.0 & 1861 & & & 79.0 \\
\hline \multirow[t]{2}{*}{24} & 69.01 & $87.86^{\circ}$ & 78. & 56.0 & is & 101.0 & " & 78.8 \\
\hline & 69.07 & $8(6.7 !)$ & 77.93 & 56.4 & & 101.1 & & 79.0 \\
\hline
\end{tabular}




\begin{tabular}{|c|c|c|c|c|c|c|c|c|}
\hline \multirow[t]{2}{*}{$1836-1882$} & \multicolumn{3}{|c|}{$\begin{array}{l}\text { Mean Values for each } \\
\text { Day. }\end{array}$} & \multicolumn{4}{|c|}{$\begin{array}{l}\text { Extreme Maxima and Minima observed } \\
\text { on each Day of the Year. }\end{array}$} & \multirow{2}{*}{$\begin{array}{l}\text { Sup. } \\
\text { posed } \\
\text { true } \\
\text { Mean }\end{array}$} \\
\hline & Min. & Max. & Mean. & Min. & Year. & Max. & Year. & \\
\hline \multirow{6}{*}{$\begin{array}{r}\text { Jul. } 25 \\
26 \\
27 \\
28 \\
29\end{array}$} & 70.24 & 88.38 & 79.31 & 59.0 & 1876 & 100.0 & 1870 & 78.6 \\
\hline & & & & & 18 & & & 78.4 \\
\hline & 70.38 & & 78. & & & 01.5 & & 782 \\
\hline & 70.23 & 87.80 & 79. & 56.0 & "6 & 00 & 1838 & 78.0 \\
\hline & 70.58 & 87.58 & 79. & 61.0 & 1853,1865 & 101.0 & 1854 & 78.0 \\
\hline & & 87.92 & 79. & 58.0 & & 100.4 & & 78.2 \\
\hline 30 & 68.52 & | 85.57 & 77. & & 1847 & & 1838 & 77.8 \\
\hline 31 & 67.96 & 86.47 & 77 & 5 & 18 & 1 & 18 & 77.8 \\
\hline \multirow{2}{*}{ Aug. 1} & 68.38 & 87.33 & 77. & 52 & 1842 & 101.0 & & 77.5 \\
\hline & & 86.95 & 77. & 52 & & 99.0 & 1861 & 77.5 \\
\hline \multirow[t]{2}{*}{3} & 68.61 & 86.59 & 77. & 55 & "“ & $\mid 101.0$ & . & 77.5 \\
\hline & 68.34 & 86.58 & 77. & 53.8 & & 100.4 & & 77.6 \\
\hline 4 & 68.40 & 85.51 & & 54. & 1880 & 101.5 & 18 & 77.5 \\
\hline 5 & 68 & 86.01 & 77 & & & 97.5 & & 77.5 \\
\hline 6 & 6 & 87 & 78 & 58 & 1842 & 98 & 1838 & 77.5 \\
\hline 7 & 69.11 & 87.07 & & & & 10 & & 77.4 \\
\hline \multirow[t]{2}{*}{8} & 68.96 & 87.09 & 78 & & 1869 & & 1850 & 77.4 \\
\hline & 68.79 & 86.57 & 77. & 56.7 & & 3 & & 77.4 \\
\hline 9 & 68.94 & 85.14 & 77. & 58 & 1879 & 10 & 1881 & 77.4 \\
\hline 10 & 69.07 & & & & & & & 77.4 \\
\hline \multirow{2}{*}{$\begin{array}{l}11 \\
12\end{array}$} & 6 & 4 & 7 & & 6 & 10 & & 77.4 \\
\hline & 68.38 & 86.47 & 77 & 36 & 1868 & 10 & & 77.2 \\
\hline \multirow[t]{2}{*}{13} & 68.72 & 86.08 & & & 1860 & 10 & 1850 & 77.0 \\
\hline & 68. & 85.50 & 38 & 5 & & 10 & & 77.3 \\
\hline \multirow{2}{*}{$\begin{array}{l}14 \\
15\end{array}$} & 68. & 85.30 & 76 & 5 & 180 & 98.5 & & 77.0 \\
\hline & & 85. & & & & & & 76.9 \\
\hline 16 & 67. & 86.22 & & & 18 & & & 76.8 \\
\hline 17 & 67. & 85.54 & 76 & 54 & 18 & 97.0 & 1843,1860 & 76.7 \\
\hline 18 & 68.03 & 86.27 & 77 & 56.0 & 185.5, & 99.5 & 1850 & 76.5 \\
\hline & 68.09 & 85.74 & & 56.6 & & 97.6 & & 76.8 \\
\hline 19 & 68.19 & 85.25 & & & 18 & 9 & 18 & 76.4 \\
\hline 20 & 66 & 85. & & & & & & 76.3 \\
\hline 21 & 67. & & & & & & 18 & 76.2 \\
\hline 22 & 67. & 8 & & & & & & 76.0 \\
\hline 23 & 66.3 & 84.09 & 75. & 52.0 & 1866 & 97.5 & 1869 & 76.0 \\
\hline & & 85. & 76. & 54.0 & & & & 76.2 \\
\hline 24 & 66 & 85.29 & & & 1866 & & $18 \div 2$ & 75.8 \\
\hline 25 & 66 & 84. & 75 & 5( & & 10 & & 75.5 \\
\hline 26 & 67. & 84. & & & & & 6 & 75.3 \\
\hline 27 & 65. & 84. & & & & & 183 & \\
\hline 28 & 65.37 & 82.98 & 74. & 51 & 1844 & 97.5 & 1881 & 74.8 \\
\hline & 66.25 & 84.35 & & 50 & & 98.1 & & 75.3 \\
\hline 29 & 64 & 83.05 & & & 10 & & & 4.5 \\
\hline 30 & & & & & & & & 74.3 \\
\hline 31 & & & & 48 & & 99.0 & 1854,1873 & 74.2 \\
\hline ept. 1 & 64.75 & 83. & & 50.0 & & & 1854 & 74.0 \\
\hline 2 & 65.08 & 82.80 & 73. & 51 & 1850 & 102.0 & 1864 & 74.0 \\
\hline & 64.93 & 83.37 & & 48 & & & & 74.5 \\
\hline 3 & & 83.00 & & & & & & 4.0 \\
\hline 4 & 65.10 & & & & & & 1881 & 74.0 \\
\hline 5 & & 82. & & $5 n$ & & & 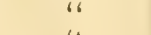 & 73. \\
\hline 6 & 64.98 & 81.84 & 73. & 51 & & 97.0 & $"$ & 73.6 \\
\hline 7 & 65.61 & 83.17 & $\begin{array}{r}74.39 \\
-\end{array}$ & 52.0 & 1849 & 97.0 & $"$ & 73.4 \\
\hline & 65.11 & 82.75 & 73.93 & 51.1 & & 99.2 & & 73.2 \\
\hline
\end{tabular}


TRANS. ST. LOUIS ACAD. SCIENCE.

\begin{tabular}{|c|c|c|c|c|c|c|c|c|}
\hline \multirow[t]{2}{*}{$1836-18 s 2$} & \multicolumn{3}{|c|}{$\begin{array}{l}\text { Mean Values for each } \\
\text { Day. }\end{array}$} & \multicolumn{4}{|c|}{$\begin{array}{l}\text { Extreme Maxima and Minima observed } \\
\text { on each Day of the Year. }\end{array}$} & \multirow{2}{*}{$\begin{array}{l}\text { Sup- } \\
\text { posed } \\
\text { true } \\
\text { Mean }\end{array}$} \\
\hline & Min. & Max. & Mean. & Min. & Year. & Max. & Year. & \\
\hline Sept. 8 & 64.48 & 83.47 & 73.97 & 49.5 & 18 & 99.5 & 4 & 73.0 \\
\hline & 63 & 33 & & & 1847,1869 & & & 72.0 \\
\hline 10 & 62.69 & 79.95 & 71. & 46 & 1880 & 96.5 & 1842 & 71.0 \\
\hline 11 & 61.01 & 78.90 & 69. & 47 & 1878 & 0 & & 70.2 \\
\hline \multirow[t]{2}{*}{12} & 60.69 & 77.79 & 69 & 46.0 & 1839,1878 & 0 & 1865 & 70.0 \\
\hline & 62.51 & 80.15 & 71.33 & 47.9 & & 1 & & 71.2 \\
\hline 12 & 60.27 & 77.85 & 69.06 & 47.0 & 1839,1878 & 98 & 1851,1864 & 70.0 \\
\hline 14 & & & & 43 & 1873 & 0 & 10 & 69.5 \\
\hline 15 & 60.51 & 77 & 69 & 47 & 15 & 88. & 1849,1862 & 69.0 \\
\hline 16 & 60.76 & 79.34 & 70 & & 18 & 91.5 & 1857 & 69.0 \\
\hline \multirow[t]{2}{*}{17} & 60.54 & 77.72 & 69. & & 1868 & 3.0 & 1843,1857 & 68.5 \\
\hline & 60.49 & 78.39 & 69. & 4 & & 7 & & 9.2 \\
\hline 18 & 6 & 78 & 6 & & ${ }_{66}^{1863}$ & & ${ }_{6}^{1867}$ & 68.0 \\
\hline 19 & & & & & & & 1881 & 67.0 \\
\hline 21 & 5. & 73 & & & & & $\begin{array}{l}1001 \\
1872\end{array}$ & 66.0 \\
\hline \multirow[t]{2}{*}{22} & 55.39 & 73.69 & 64.54 & 39 & 1875 & 12 & 1881 & 65.8 \\
\hline & 56.89 & 74.96 & 65.93 & 40 & & 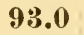 & & 66.5 \\
\hline $2 ?$ & 58.17 & 76.82 & 67 & 36 & 1856 & 92 & 1881 & 65.5 \\
\hline 24 & +58.15 & 76.23 & & 37 & & b) & \& & 65.0 \\
\hline 25 & 57.09 & 75. & & & 1879 & 5 & 1850 & 64.8 \\
\hline 26 & 54 & 73. & 64 & 40 & 18 & 92. & 1847 & 64.5 \\
\hline \multirow[t]{2}{*}{27} & 54.87 & 73.82 & 64.34 & 41 & 1871 & 88. & 1854 & 64.3 \\
\hline & & 85 & & & & & & 4.8 \\
\hline 28 & 354. & 7 & & & 1839 & 90 & $186^{\prime}$ & 4.0 \\
\hline 29 & 54.17 & 71. & 6 & 3 & 18 & 93. & 18 & 3.5 \\
\hline 30 & 52.59 & 71.53 & & & & & & 63.0 \\
\hline \multirow[t]{27}{*}{ Oct. } & 1. 54.64 & 73.37 & 64 & & 1856 & 8 & 185 & 63.0 \\
\hline & 254.68 & 73.74 & 64.21 & 38.5 & & 91 & 1867 & 63.0 \\
\hline & 54.09 & 72.81 & {$[63.45]$} & & & 9 & & 3.1 \\
\hline & 354.52 & & & & 1840 & & 1872 & 62.5 \\
\hline & 452.29 & & & 31 & 18 & 88 & 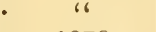 & 62.0 \\
\hline & 552.18 & 70 & 61 & 3 & 6 & 8 & 187 & 61.5 \\
\hline & $\begin{array}{l}6 \\
52.52\end{array}$ & 70 & & & 18 & & 1852 & 61.5 \\
\hline & 751.93 & 70.62 & 61. & 3 & 1873 & 87 & 1860 & 61.0 \\
\hline & 52.69 & 71.03 & 61.56 & 8 & & 88 & & 1.7 \\
\hline & 8 51.70 & 71.14 & 61 & 3 & & & 8 & 0. \\
\hline & & & & & 1842 & & 1879 & 60.0 \\
\hline & 051 & & & 3 & 18 & & "6 & 59. \\
\hline & 149.98 & 67 & & 31 & & & & 59. \\
\hline & 248.73 & 64.91 & 56.8 & 30 & 1875 & 8 & " & 58. \\
\hline & & & & & & & & 59. \\
\hline & 345 & 6 & 55 & 2 & & 81.5 & 1879 & 58. \\
\hline & 447.20 & & & & & & 1878 & 57. \\
\hline & 546.88 & & & & & & 1881 & 57. \\
\hline & $\begin{array}{ll}6 & 47.34\end{array}$ & & & 27 & & 83.0 & 1842 & 56. \\
\hline & 746.80 & 65.42 & 56. & 31.0 & 1836,1868 & 83.0 & 1839,1842 & 55. \\
\hline & 46.84 & 65.61 & 156 & 29 & & & & 56. \\
\hline & 844.64 & & & & & & 18 & 54 \\
\hline & 942 & & & 31 & & & 10 & 54 \\
\hline & $\begin{array}{ll}0 & 42.75\end{array}$ & 62.31 & 52. & 24 & 1836 & 83.0 & 1843 & 53. \\
\hline & 1) 44.60 & 62.47 & & 25.0 & & 84.0 & 1837 & 53. \\
\hline & 244 & 61.47 & 52.8 & 30.0 & 1869 & 85.0 & & 52. \\
\hline & 45 & 61. & 82 & 28.7 & & 83.7 & & \\
\hline
\end{tabular}




\begin{tabular}{|c|c|c|c|c|c|c|c|c|}
\hline \multirow[t]{2}{*}{ 1836-1882 } & \multicolumn{3}{|c|}{$\begin{array}{l}\text { Mean Values for each } \\
\text { Day. }\end{array}$} & \multicolumn{4}{|c|}{$\begin{array}{l}\text { Extreme Maxima and Minima observed } \\
\text { on each Day of the Year. }\end{array}$} & \multirow{2}{*}{$\begin{array}{l}\text { Sup- } \\
\text { posed } \\
\text { true } \\
\text { Mean }\end{array}$} \\
\hline & Min. & Max. & Mean. & Min. & Year. & Max. & Year. & \\
\hline Oct. 23 & $\overline{43.41}$ & 61.54 & 52.47 & 20.0 & 1863 & 79.5 & 1839 & 52.0 \\
\hline & 44.32 & 60.64 & 52.48 & & & & & \\
\hline 25 & 42.51 & $\begin{array}{l}59.96 \\
\end{array}$ & 51.23 & 22.0 & 1841 & 78.5 & 1882 & 51. \\
\hline 26 & 43.64 & 60.56 & 52.10 & 21.5 & 1862 & 81.5 & 1874 & 1.4 \\
\hline \multirow[t]{2}{*}{27} & 42.15 & 61.11 & 51.63 & 26.0 & 1869 & 81.5 & 1870 & 51. \\
\hline & 43.20 & 60.76 & 51.95 & 21.9 & & 79.8 & & 1.8 \\
\hline 28 & 43.54 & 60.56 & 52.05 & 27.0 & 1873,1878 & 80.5 & 1874 & 51. \\
\hline 29 & 43.71 & 60.77 & 52.24 & 22.0 & 1873 & 81.5 & & 50. \\
\hline 30 & 42.34 & 57.28 & 49.81 & 26.5 & 1863 & 81.5 & 1876 & 50. \\
\hline 31 & 39.75 & 56.66 & 48.20 & 19.5 & $" 6$ & 80.0 & 66 & 50. \\
\hline \multirow{2}{*}{ Nov. 1} & 42.01 & 59.97 & 50.99 & 22.0 & 1873 & 78.0 & 1842,1876 & 50. \\
\hline & 42.27 & 59.05 & 50.66 & 23.4 & & S0.5 & & 50.4 \\
\hline 2 & 43.20 & 58.00 & 50.60 & 30.5 & 1848 & 77.0 & 1847 & 50. \\
\hline 3 & 41.11 & 56.59 & 48 & 2 & & & & 49. \\
\hline 4 & 42.01 & 58.66 & 50. & 23 & & 80.0 & 1850 & 49. \\
\hline 5 & 41.23 & 55.70 & 48.46 & 25.0 & 1865 & 72.5 & 1874 & 49. \\
\hline \multirow[t]{2}{*}{6} & 38.91 & 55.29 & 47. & 23 & & 73.0 & 1874,1878 & 48. \\
\hline & +1.29 & 56.85 & 19. & 25.5 & & 75.6 & & 49.3 \\
\hline 7 & 40.78 & 57.55 & 49.16 & 24.0 & 1856 & 77.0 & 1874 & 48. \\
\hline 8 & 40.30 & 55.78 & & & & & & 7.0 \\
\hline 9 & 37. & 51. & & & . & & & 46 \\
\hline 10 & 39.03 & 52.71 & 45. & 20 & 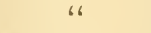 & 76.0 & & 45.5 \\
\hline \multirow[t]{2}{*}{11} & 40.23 & 53.53 & 46. & 26.5 & 1869 & 81 & 1837 & 45.0 \\
\hline & 39.5 .5 & 54.89 & 46.92 & 19.5 & & 77.0 & & 46.3 \\
\hline 12 & 36.93 & 51.66 & 44.29 & 17 & 1859 & 71 & 18 & 44. \\
\hline 13 & 36.66 & 52.82 & 44. & & & 79 & & $4 .($ \\
\hline 14 & 36.67 & 51.86 & & 20 & 18 & & & 3 \\
\hline 15 & 35.18 & 50.01 & & & & 7 & & 43.0 \\
\hline & 36.11 & 49.60 & 42.85 & 10.0 & 10 & 69.0 & 1865 & 42.0 \\
\hline & 36.31 & 51.19 & 48.75 & 16.0 & & 72.6 & & 43.4 \\
\hline 17 & 35.55 & 47.80 & 41.67 & 12.0 & 18 & 69 & 18 & 41.0 \\
\hline 18 & 33.62 & 45.58 & 39 & 6 & 18 & 72 & & 40.5 \\
\hline 19 & 31.60 & & & & ' & 7 & & 40.0 \\
\hline 20 & 32.59 & 45.11 & $3 s$ & & 18 & 72 & 183 & 39.5 \\
\hline \multirow[t]{2}{*}{21} & 33.09 & 45.84 & 39.46 & 10.5 & 1880 & 69.0 & 1841 & 39.0 \\
\hline & 33.09 & 4.5 .57 & 39.55 & 9.1 & & 70.7 & & 40.0 \\
\hline 22 & 32.28 & 45.92 & 39 & 6 & 18 & 71 & 10 & $3 S$. \\
\hline 23 & 32.14 & 43.97 & $3 s$ & 9 & & $6 ?$ & & 38. \\
\hline 24 & 29.85 & 41.93 & 35 & 5. & & & & 37. \\
\hline 25 & 29.83 & $41.9^{2} 2$ & 35 & & & & 18 & 37. \\
\hline \multirow[t]{2}{*}{6} & 31.25 & 44.06 & 37.65 & 14.0 & is & 65.11 & 1850 & 37.4 \\
\hline & 31.07 & 43.56 & 37.31 & 7.1 & & 67.0 & & 37.6 \\
\hline 27 & 31.44 & 43.22 & & 5.0 & 184 & 67 & 187 & $3 \pi$. \\
\hline 28 & 31.64 & 44.05 & 37 & 0 & " & 72 & 18 & 36. \\
\hline 29 & 29.04 & 42.76 & 35. & 2.0 & 187 & 76.5 & & 36. \\
\hline & $28.5 i$ & 4.2 .98 & 35. & 6.5 & & 72.5 & 1837 & 36. \\
\hline \multirow[t]{8}{*}{ Dec. 1} & 30.33 & 45.57 & 37.95 & -1.0 & ‘. & 72.5 & 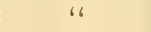 & 36. \\
\hline & 30.20 & 43.71 & $3(6.95$ & 2.4 & & $7 \because .1$ & & 36. \\
\hline & 31.06 & $\$ 4.95$ & 38.110 & 4.0 & 18 & 72.0 & 1864 & 35 . \\
\hline & 30.61 & 43.00 & & 8. & 18 & $\$ 1.0$ & 1842,1873 & 35. \\
\hline & 30.75 & 42.18 & 36.46 & - 2.5 & 18 & 59.0 & 1877 & 35. \\
\hline & 29.45 & 41. & 35. & 2.5 & & 61.0 & & - \\
\hline & 29.56 & 41.58 & 35.72 & 3.5 & 1859 & 62.0 & 1861 & 35. \\
\hline & 30.25 & 42.67 & $: 36.47$ & 3.1 & & 63.0 & & 35. \\
\hline
\end{tabular}




\begin{tabular}{|c|c|c|c|c|c|c|c|c|}
\hline \multirow{2}{*}{$1836 \cdot 1882$} & \multicolumn{3}{|c|}{$\begin{array}{l}\text { Mean Values for each } \\
\text { Day. }\end{array}$} & \multicolumn{4}{|c|}{$\begin{array}{l}\text { Extreme Maxima and Minima observed } \\
\text { on each Day of the Year. }\end{array}$} & \multirow{2}{*}{$\begin{array}{l}\text { Sup- } \\
\text { posed } \\
\text { true } \\
\text { Mean }\end{array}$} \\
\hline & Min. & Max. & Mean. & Min. & Year. & Max. & Year. & \\
\hline \multirow[t]{30}{*}{ Déc. } & 28.00 & 40.23 & 34.11 & -11.0 & 188 & 63.0 & 1851 & 34.0 \\
\hline & 27.79 & 39.11 & 3 & -4.0 & & 65.0 & & \\
\hline & 25.07 & 35.35 & 30.21 & -5.0 & 1876 & 74.5 & & 33.0 \\
\hline & 23.89 & 36.06 & 29.97 & 0.0 & 1868 & 68.0 & " & 32.5 \\
\hline & 28.19 & 37.61 & 32.90 & -11.0 & $" 6$ & 68.0 & 1873 & 32.0 \\
\hline & 06.59 & 37.67 & 32.13 & -6.2 & & 67.7 & & 33.0 \\
\hline & 25.54 & 37.15 & 31.34 & -0.5 & 1868 & 68.5 & 1877 & 31.8 \\
\hline & 26.25 & 36.70 & 31.47 & 4.0 & 1865 & 62.5 & 1 & 31.6 \\
\hline & 23.46 & 33.79 & 28.62 & -1.0 & & 59.0 & 1861 & 31.4 \\
\hline & 23.57 & 33.46 & 28.51 & -2.5 & 1851 & 67.5 & 1877 & 31.2 \\
\hline & 24.29 & 34.83 & 29.56 & -0.5 & "6 & 62.0 & & 31.0 \\
\hline & 24.62 & 35.18 & 29.90 & -0.1 & & 63.9 & & 31.4 \\
\hline & 25.10 & 36.91 & 31.00 & -2.5 & 1875 & 67.0 & 1877 & 31.0 \\
\hline & 25.76 & 38.87 & 32. & 1.0 & 1876 & 69.0 & "6 & 30.8 \\
\hline & 26.37 & 3810 & 32.23 & 0.5 & 1863 & 67.5 & ، & 30.6 \\
\hline & 2351 & 35.84 & 29.67 & -2.0 & 1871 & 65.0 & "6 & 30.4 \\
\hline & 24.84 & 36.97 & 30.90 & -2.0 & 1865 & 66.0 & 16 & 30.0 \\
\hline & 25.11 & 37.34 & 31.22 & -1.0 & & 66.9 & & 30.5 \\
\hline & 22.64 & 33.86 & 28.25 & -14.0 & 1872 & 62.0 & 1877 & 30.0 \\
\hline & 22.51 & 35.34 & 28.92 & -7.0 & 1870 & 61.0 & 1875 & 30.2 \\
\hline & 23.90 & 36.62 & 30 & -19.5 & 1872 & 66.0 & 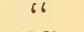 & 30.5 \\
\hline & 25.42 & 37.95 & 31.68 & -11.0 & "“ & 70.0 & 186 & 31.0 \\
\hline & 25.63 & 38.06 & 31.84 & -5.0 & " & 65.0 & 1875 & 31.2 \\
\hline & 24.0 & 36.36 & & -11.3 & & 64.5 & & 30.6 \\
\hline & 26.47 & 38.41 & 32.44 & -6.0 & 1872 & 65.0 & 184 & 31.3 \\
\hline & 26.34 & $37.81)$ & 32.0 & -6.0 & 1880 & 59.0 & 1862 & 31.5 \\
\hline & 25.83 & 37.49 & 31.66 & -18.0 & "6 & 64.0 & 1851 & 31.5 \\
\hline & 23.26 & 36.73 & 29.99 & -8.0 & "6 & 66.0 & 1875 & 31.5 \\
\hline & 24.25 & 38.43 & 31.94 & -10.5 & 1863 & 73.5 & 16 & 31.5 \\
\hline & 25 & 36.67 & & -9.7 & & 60.5 & & 11.5 \\
\hline
\end{tabular}

The annexed diagram, for the construction of which I am indebted to Dr. G. Hambach, represents the principal results of these tables. The perpendicular lines divide the year into 73 periods of 5 days each, while the horizontal ones mark the degrees. The central full-faced curve indicates the Mean Temperature of the penthemeral periods of the 47 years, as actually found, while the dotted line represents the supposed real Mean Temperature as suggested in the last column of these tables. The uppermost curve shows the Highest Temperatures and the lowest curve the Lowest Temperatures observed in those same penthemeral periods within the sime number of years. It will be noticed that while the points of Mean Temperature occupy the centre of each period, the Maxima and Minima do not show in the middle of the spaces, but on that one of the 5 days of the period on which they actually did occur. 






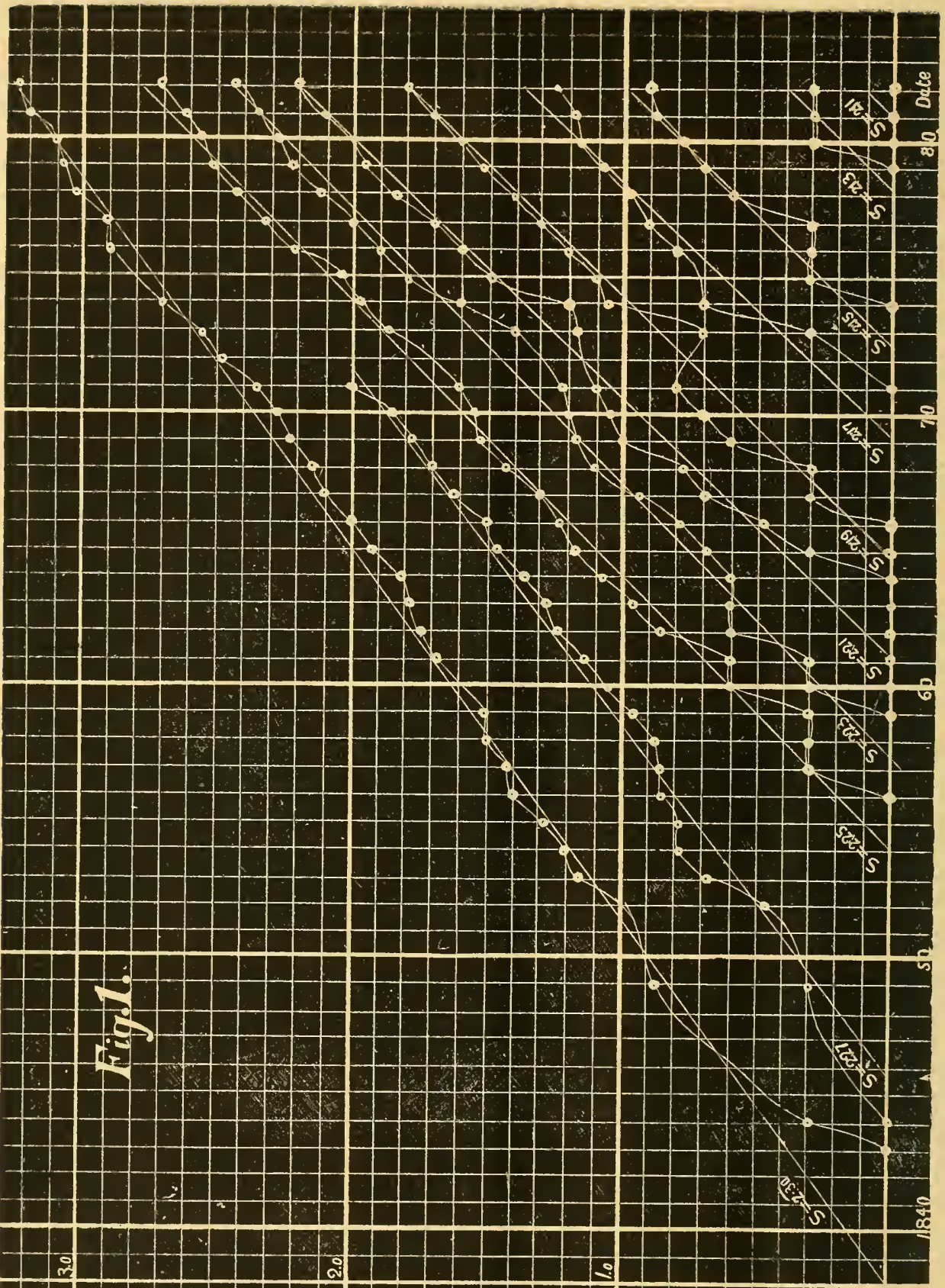




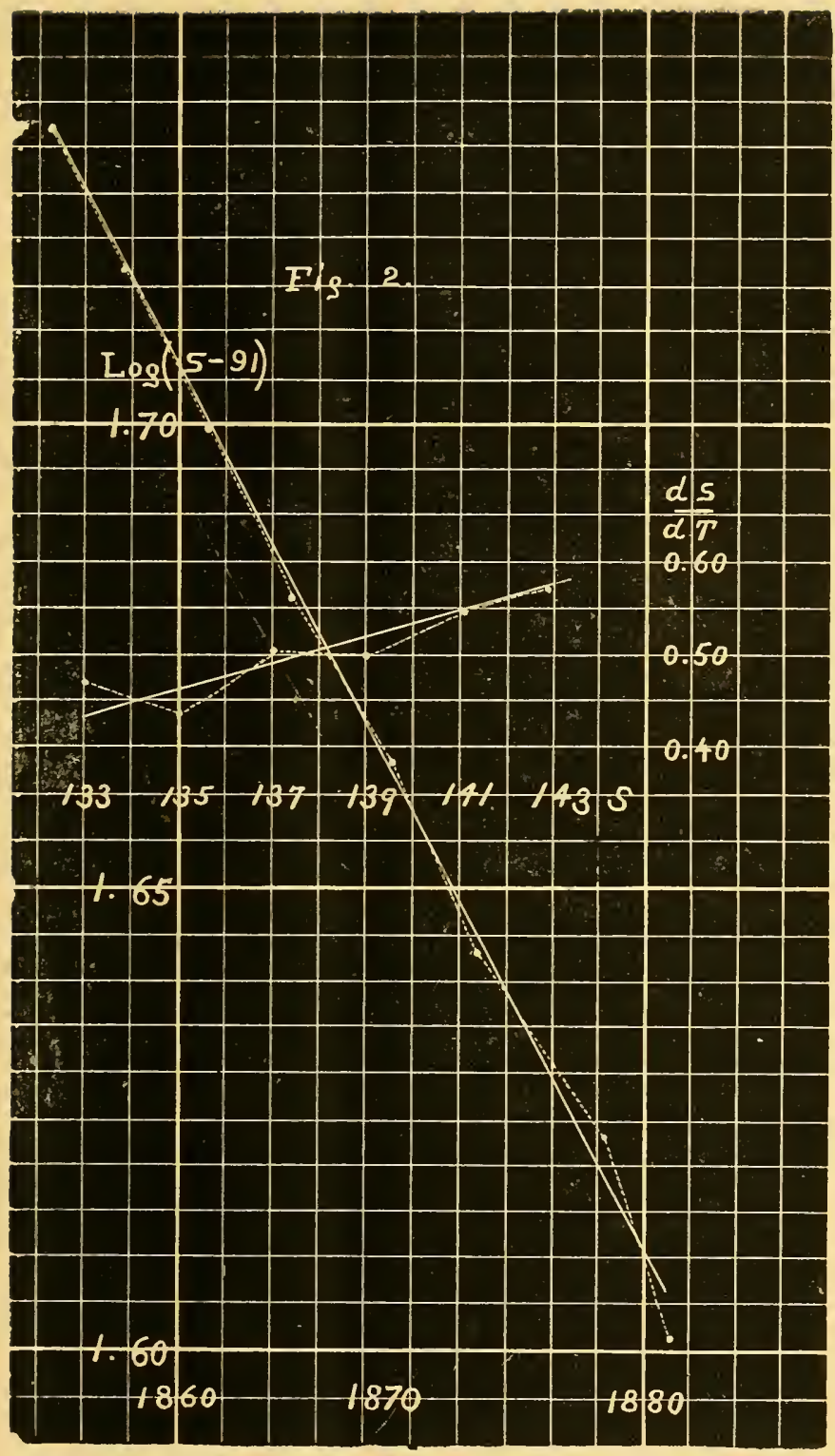





\section{The Evolution of the American Trotting.Horse.*}

By Frincis E. Nipher.

In the American Journal of Science for April, i 883, Prof. W. $H$. Brewer has furnished data for the discussion of the change in speed of the American trotting-horse. His table is here reproduced.

TABLE SHOWING THE NUMBER OF HORSES UNDER THE RESPECTIVE RECORDS.

\begin{tabular}{|c|c|c|c|c|c|c|c|c|c|c|c|}
\hline & Year. & $\begin{array}{c}2: 30 \\
\text { or } \\
\text { better }\end{array}$ & $\left|\begin{array}{c}2: 27 \\
\text { or } \\
\text { better }\end{array}\right|$ & $\begin{array}{c}2: 25 \\
\text { or } \\
\text { better }\end{array}$ & $\mid \begin{array}{c}2: 23 \\
\text { or } \\
\text { better }\end{array}$ & $\begin{array}{c}2: 21 \\
\text { or } \\
\text { better }\end{array}$ & $\begin{array}{c}2: 19 \\
\text { or } \\
\text { better }\end{array}$ & $\begin{array}{c}2: 17 \\
\text { or } \\
\text { better }\end{array}$ & $\begin{array}{c}2: 15 \\
\text { or } \\
\text { better }\end{array}$ & $\begin{array}{c}2: 13 \\
\text { or } \\
\text { better }\end{array}$ & $\begin{array}{c}2: 11 \\
\text { or } \\
\text { better }\end{array}$ \\
\hline 43 & 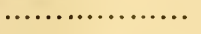 & 1 & 1 & $\cdots \cdots \cdots$ & $\cdots \cdots \cdots$ & $\cdots \cdots \cdots$ & $\cdots \cdots$ & $\cdots \cdots$ & $\cdots \cdots$ & $\cdots \cdots$ & $\cdots \cdots$ \\
\hline 1844 & $\cdots \ldots \ldots \ldots \ldots \ldots$ & 2 & 1 & $\ldots \ldots$ & $\ldots \ldots$ & $\ldots \ldots$ & $\cdots \ldots$ & $\cdots \cdots$ & $\cdots \cdots$ & $\cdots \cdots$ & $\ldots \ldots$ \\
\hline 1849 & $\ldots \ldots \ldots \ldots \ldots \ldots$ & 7 & 2 & $\cdots \cdots$ & $\cdots \cdots$ & $\cdots \cdots$ & $\cdots \cdots$ & $\cdots \cdots$ & $\cdots \cdots$ & $\cdots \cdots$ & $\cdots \cdots$ \\
\hline 1852 & $\ldots \ldots \ldots \ldots \ldots \ldots$ & 10 & 3 & $\ldots \ldots$ & $\ldots \ldots$ & $\ldots \ldots$ & $\cdots \cdots$ & $\cdots \cdots$ & $\cdots \cdots$ & $\cdots$ & $\cdots \cdots$ \\
\hline 1853 & $\ldots \ldots \ldots \ldots \ldots \ldots$ & 14 & 5 & $\cdots \cdots$ & $\cdots \cdots$ & $\cdots \cdots$ & $\cdots \cdots$ & $\cdots \cdots$ & $\cdots \cdots$ & $\cdots$ & $\cdots \cdots$ \\
\hline 54 & $\ldots \ldots \ldots \ldots \ldots$ & 16 & 6 & $\ldots \ldots$ & $\cdots \cdots$ & $\cdots \cdots$ & $\cdots \cdots$ & $\cdots \cdots$ & $\ldots \ldots$ & $\cdots$ & ...... \\
\hline 1855 & $\ldots \ldots \ldots \ldots \ldots \ldots$ & 19 & 6 & $\cdots \ldots$ & $\cdots \cdots$ & $\cdots \cdots$ & $\cdots \cdots$ & $\cdots \cdots$ & $\cdots \cdots$ & $\cdots$ & $\cdots \cdots$ \\
\hline 1856 & $\ldots \ldots \ldots \ldots \ldots \ldots$ & 24 & 7 & 1 & $\cdots \cdots$ & $\ldots \ldots$ & $\ldots \ldots$ & $\cdots \cdots$ & $\cdots \cdots$ & $\cdots \cdots$ & $\ldots \ldots$ \\
\hline 1857 & $\ldots \ldots \ldots \ldots \ldots \ldots$ & 26 & 7 & 2 & $\cdots \cdots \cdots$ & $\cdots \cdots$ & $\cdots \cdots$ & $\cdots$ & $\cdots \cdots$ & $\cdots \cdots$ & $\cdots \cdots$ \\
\hline 1858 & $\ldots \ldots \ldots \ldots \ldots \ldots$ & 30 & 7 & 2 & $\cdots \cdots$ & $\cdots \cdots$ & $\cdots \cdots$ & $\cdots \cdots$ & $\cdots \cdots$ & $\cdots \cdots$ & $\ldots \ldots$ \\
\hline 59 & $\ldots \ldots \ldots \ldots$ & 32 & 9 & 2 & 1 & 1 & $\ldots \ldots$ & $\cdots \cdots$ & $\cdots \cdots$ & $\cdots \cdots \cdots$ & $\ldots \ldots$ \\
\hline 60 & $\ldots \ldots \ldots \ldots \ldots \ldots$ & 40 & 11 & 4 & 2 & 1 & $\cdots \ldots$ & $\cdots \cdots$ & $\cdots \cdots$ & $\cdots \cdots$ & $\ldots \ldots$ \\
\hline 31 & $\ldots \ldots \ldots \ldots \ldots \ldots$ & 48 & 14 & 4 & 2 & 1 & $\ldots \ldots$ & $\cdots \ldots$ & $\cdots \cdots$ & $\ldots \ldots$ & $\ldots \ldots$ \\
\hline 52 & $\ldots \ldots \ldots \ldots \ldots \ldots$ & 54 & 17 & 7 & 4 & 1 & $\ldots \ldots$ & $\ldots \ldots$ & $\cdots \cdots$ & $\ldots \ldots$ & $\ldots \ldots$ \\
\hline 1863 & $\ldots \ldots \ldots \ldots \ldots$ & 59 & 19 & 9 & 4 & 1 & $\ldots \ldots$ & $\ldots \ldots$ & $\cdots \cdots$ & $\ldots \ldots$ & $\cdot \cdots$ \\
\hline 1864 & $\ldots \ldots \ldots \ldots \ldots$ & 66 & 22 & 12 & 4 & 1 & $\cdots$ & $\ldots \ldots$ & $\ldots \ldots$ & $\ldots \ldots$ & $\ldots \ldots$ \\
\hline 1865 & $\ldots \ldots \ldots \ldots \ldots \ldots$ & 81 & 29 & 15 & 5 & 2 & 1 & $\ldots \ldots$ & $\ldots \ldots$ & $\ldots \ldots$ & $\ldots \ldots$ \\
\hline 1866 & $\ldots \ldots \ldots \ldots \ldots \ldots$ & 101 & 32 & 17 & 6 & 3 & 1 & $\ldots \ldots$ & $\ldots \ldots$ & $\ldots \ldots$ & $\ldots \ldots$ \\
\hline 1867 & $\ldots \ldots \ldots \ldots \ldots \ldots$ & 124 & 42 & 21 & 9 & 5 & 2 & $\ldots \ldots$ & $\ldots \ldots$ & $\ldots \ldots$ & $\ldots \ldots$ \\
\hline 1868 & $\ldots \ldots \ldots \ldots \ldots \ldots \ldots$ & 146 & 52 & 28 & 13 & 6 & 2 & . & $\ldots \ldots$ & .. & $\ldots \ldots$ \\
\hline 1869 & $\ldots \ldots \ldots \ldots \ldots \ldots \ldots$ & 171 & 63 & 34 & 15 & 10 & 4 & $\ldots \ldots$ & $\ldots \ldots$ & . & $\ldots \ldots$ \\
\hline 1870 & $\ldots \ldots \ldots \ldots \ldots \ldots$ & 194 & 72 & 35 & 16 & 11 & 5 & $\ldots \ldots$ & . & .. & $\ldots \ldots$ \\
\hline 1871 & $\ldots \ldots \ldots \ldots \ldots \ldots$ & 23.3 & 99 & 40 & 17 & 12 & 6 & 1 & $\ldots \ldots$ & $\ldots \ldots$ & $\ldots \ldots$ \\
\hline 1872 & $\ldots \ldots \ldots \ldots \ldots$ & 323 & (..... & ?. & $\cdots$ & $\because \cdots$ & $\cdots$ & $\ldots \ldots$ & $\cdots \cdots$ & $\ldots \ldots$ & $\ldots \ldots$ \\
\hline 1873 & $\ldots \ldots \ldots \ldots$ & 376 & $\ldots \ldots$ & 74 & 28 & 15 & 5 & 2 & $\ldots \ldots$ & $\ldots \ldots$ & $\ldots \ldots$ \\
\hline 1574 & $\ldots \ldots \ldots \ldots$ & 506 & $\cdots$ & 98 & 40 & 16 & 11 & 5 & 1 & $\ldots \ldots$ & $\ldots \ldots$ \\
\hline 1875 & $\ldots \ldots \ldots \ldots \ldots \ldots$ & .... & $\cdots \ldots$ & 134 & 61 & 30 & 13 & 5 & 2 & $\ldots \ldots$ & $\ldots \ldots$ \\
\hline 1876 & …........ & 791 & $\cdots$ & 16.5 & 81 & 39 & 16 & 6 & 2 & $\cdots$ & $\ldots \ldots$ \\
\hline 1877 & $\ldots \ldots \ldots \ldots \ldots$ & 836 & $\ldots \ldots$ & 214 & 105 & 51 & 19 & 8 & 2 & - & $\ldots \ldots$ \\
\hline 1878 & $\ldots \ldots \ldots \ldots \ldots \ldots$ & 1025 & $\ldots \ldots$ & 270 & 129 & 68 & 24 & 9 & 4 & & \\
\hline 1879 & $\ldots \ldots \ldots \ldots \ldots \ldots$ & 1142 & $\ldots \ldots$ & 325 & 164 & 88 & 33 & 11 & 5 & 1 & ... \\
\hline 1880 & $\ldots \ldots \ldots \ldots \ldots$ & 1210 & $\ldots \ldots$ & 366 & 192 & 106 & 41 & 14 & 6 & 2 & 1 \\
\hline 1881 & $\ldots \ldots \ldots \ldots \ldots \ldots$ & 1532 & $\ldots \ldots$ & 419 & 227 & 126 & 49 & 15 & 7 & 2 & 1 \\
\hline 1882 & $\ldots \ldots \ldots \ldots \ldots \ldots$ & 1684 & $\ldots \ldots$ & 495 & 275 & 156 & 60 & 18 & 8 & 2 & 1 \\
\hline
\end{tabular}

Prof. Brewer states that the data for the speeds $2: 30$ and $2: 27$ are very unsatisfactory, but for all the others are reasonably correct.

On taking the logarithms of all the numbers $N$ of horses capable of trotting a mile in $s$ seconds, it results that the plotted values of $\log$. $N$ for their proper dates give a straight line for each value of $s$. For the speeds $s$ of $2: 30$ and $2: 27$ these lines are parallel to each other, and the lines representing the remaining 
speeds are also parallel to each other, but the two groups are not parallel.

For the first set, the lines can be represented by the equation

$$
\log N=A^{\prime}+0_{075} T
$$

where $A^{\prime}$ is a function of $s$, and $T$ is estimated in years from any arbitrarily assumed date.

For the second group,

$$
\log N=A^{\prime}+\text { o. }_{10} T \text {. }
$$

It is apparent that for the speed of $2: 30$ and $2: 27$ the values of $N$ are too small, for the reason perhaps that in earlier years, when this was called good time, less general attention was paid to the breeding of trotters, while in later years, as this became a common speed, a constantly increasing number of horses of this grade have been used as roadsters and remained undiscovered in private hands.

These plotted lines are shown in Fig. I. It is clear that the intersection of any one of these lines with the time axis determines the date when this speed may be supposed to have originated, or when $N=$ I, and that this determination of the date, based as it is upon a number of observations running through a series of years, is much more reliable than the date when some accidentally arranged trotting match revealed the fact that the horse capable of making this speed had already come.

The dates for the origin of the speeds of $2: 13$ and 2 : I I cannot yet be determined very accirately, and this fact is to be remembered in considering the discussion which follows.

The following table gives the values of $s$ in seconds and the dates for the origin of these speeds, determined as before explained. The third column contains the change in speed per year, calculated in a well known manner from alternate differences in the two previous columns.

\begin{tabular}{|c|c|c|}
\hline$s$. & YEAR. & $\frac{d s}{d T}$ \\
\hline I45 & $1854 \cdot 0$ & $\ldots \ldots$ \\
I43 & $1857 \cdot 4$ & 0.571 \\
I4I & I86I.0. & 0.547 \\
139 & $1864 \cdot 7$ & 0.500 \\
137 & $1869 \cdot 0$ & 0.506 \\
135 & IS72.6 & 0.430 \\
I33 & $1878 \cdot 3$ & 0.476 \\
I3I & I8SI.0 & $\cdots$ \\
\hline
\end{tabular}


When the values of $\frac{d s}{d T}$ are plotted with the simultaneous values of $s$, we get a somewhat irregular series of points shown in Fig 2, and represented fairly well by the equation

$$
\frac{d s}{d T}=A+B s \quad-\quad \quad-\quad \quad-
$$

The constants $A$ and $B$ can be determined graphically with as great precision as the nature of the data will warrant.

The values are found to be

$$
\begin{aligned}
& A=-1.00 \\
& B=+0.0110
\end{aligned}
$$

and the differential equation (I) becomes

$$
\frac{d s}{d T}=-\mathrm{I}_{000}+0.0110 \mathrm{~s} \quad-
$$

This equation being put into the form

$$
\frac{d s}{s-90.9}=0.0110 d T
$$

it admits of direct integration as follows,

$$
\int_{s_{0}-90.9}^{s}=0.0110 \int_{T}^{T_{0}}
$$

on performing the indicated operations

$$
l(s-90.9)=l\left(s_{0}-90.9\right)+0.0110 T_{0}-0.0110 \mathrm{~T}
$$

where $s_{0}$ and $T_{0}$ are simultineous values at any assumed date.

Placing the initial values in a single term, we have

$$
l(s-90.9)=C-B T
$$

or for the primitive equation

$$
s=90.9+\mathrm{e}^{C-B T}
$$

where $\mathrm{e}$ is the Naperian base.

It thus appears that the limiting speed of which the trottinghorse is capable, which he will continually approximate and never reach, is $\mathrm{I}: 3 \mathrm{I}$. This follows from (2) by making $\frac{d s}{d T}=\mathrm{o}$, or from (3) and (4) by making $T=\infty$. 
The constants $B$ and $C$ are best determined by taking the logarithms of $(s-9 \mathrm{I})$ for the valious values of $s$, and plotting them on the time axis. These values are given in the following table :

\begin{tabular}{|c|c|c|c|c|c|c|}
$s$. & $s-91$ & $\log (s-91)$ & YEAR. & $T$. & $s$ calc. & DIFF. \\
\hdashline 145 & 54 & 1.732 & 1854.0 & -6.0 & I 44.8 & -0.2 \\
143 & 52 & 1.716 & 1857.4 & -2.6 & 142.9 & -0.1 \\
141 & 50 & 1.699 & 1861.0 & +1.0 & 140.9 & -0.1 \\
139 & 45 & 1.681 & 1864.7 & -4.7 & 139.2 & +0.2 \\
137 & +6 & 1.663 & 1869.0 & -9.0 & 137.2 & +0.2 \\
135 & 44 & 1.643 & 1872.6 & +12.6 & 135.2 & +0.2 \\
133 & 42 & 1.623 & 1878.3 & +18.3 & 132.6 & -0.4 \\
131 & 40 & 1.602 & 1881.0 & +21.0 & 131.4 & +0.4 \\
\hline
\end{tabular}

The constants are determined by well known graphical methods, and it is thus found that the observations are represented by the equation

$$
\log (s-9 \mathrm{I})=\mathrm{I.703}_{100046} T,
$$

where the logarithms are common, and $T$ is estimated in years from I 860. Substituting in (4) the values of $T$ for the dates of the above table, the values of $s$ and their differences from the observed values of $s$ have been determined and are given in the final column. These differences are seen to be greatest for the later dates, where the possible errors are known to be greatest; but the error in $s$ even here cornesponds to an error of only a year in date, which is certainly within the error of observation.

From (5) it is easy to determine the date when the horse will have reached within one second of the limiting speed. Making $s=92$, this value of $T$ turns out to be 37 o years. By the close of the present century the time of trotting a mile will be reduced to $2: 04$, and the time of $2: 00$ will be reached in the year I9I2. But they indicate that the trotting-horse will finally be able to make his mile in a time not differing materially from the time of the running-horse, which is at present about roo seconds. Whether or not the trotting-horse will finally beat the runninghorse, as the present results seem to indicate, it is perhaps not possible to decide at present with the insufficient data at our command. A weighty consideration is found in the fact that a well trained trotter carries his body more steadily, or with less of rise and fall, than the racer, and this may possibly result to the 
final advantage of the trotting-horse after the process of developing and adjusting of his muscles and chest shall have been sufficiently carried on, so that the contest between the trotter and the racer shall have been reduced to a matter of muscular capacity.

It is well known that some herds of wild horses on the Texas plains were natural pacers, and even when pushed to the utmost, and for days together, by the best running-horses, the greater portion of them held to their gait. One large white pacer became widely known and his capture was often attempted, but he always proved more than a match for the best horses that could be brought against him.

Whatever may be said about the particular numerical results of this discussion, it is clear that the trotting-horse is very likely to reach a much higher speed than has been heretofore thought póssible.

\section{Added Nov. 7, 1883 .}

In the November number of the American Journal of Science Mr. W. H. Pickering has criticised the method of reduction used in the present paper (which had been printed from advance sheets in the July number of that journal), and has reached a very different conclusion from that reached in the present paper.

Mr. Pickering thinks that it is objectionable to determine the value of $\frac{d s}{d T}$ by taking the alternate differences in $s$ and $T$, and he has reduced the observations by taking differences between consecutive values in the table. In this way he gets the values of the third column in the table below.

\begin{tabular}{|c|c|c|c|c|}
\hline s. & YEAR. & $\begin{array}{r}d s \\
-d T\end{array}$ & $\frac{d s}{d T}$ calc. & $e$. \\
\hline 145 & 1854.0 & & & \\
\hline 143 & ${ }^{I S} S_{57} \cdot 4$ & 0.59 & 0.59 & 0.0 \\
\hline 141 & IS6I.O & $\begin{array}{l}0.55 \\
0.54\end{array}$ & 0.56 & - 0.1 \\
\hline I39 & IS64.7 & $\begin{array}{l}0.34 \\
0.46\end{array}$ & $\begin{array}{l}0.54 \\
0.51\end{array}$ & $\begin{array}{r}0.0 \\
-0.4\end{array}$ \\
\hline 137 & $1869 \cdot 0$ & 0.55 & 0.49 & +0.4 \\
\hline I35 & I 872.6 & 0.35 & 0.46 & -1.4 \\
\hline $\begin{array}{l}133 \\
131\end{array}$ & $\begin{array}{l}\mathbf{1} 878.3 \\
1881.0\end{array}$ & 0.7 .5 & 0.44 & +1.8 \\
\hline
\end{tabular}

Plotting these values of $\frac{d s}{d T}$ and the corresponding values of $s$, he then goes on to say that the points so determined may be represented by a curve, such that the valúe of $\frac{d s}{d T}$ increases as $s$ 
diminishes, and thus indicating disturbing causes not easily discussed. Assuming that a straight line will represent the values, he determines the value of the constants, and finds that the line intersects the axis of $s$ at a point where the value of $s$ is -25 . This would mean that the limiting speed of the trotter is $25 \mathrm{sec}-$ onds less than no time at all.

When making his first discussion of the subject, the writer considered the propriety of determining the value of $\frac{d s}{d T}$ by means of consecutive differences, and unfortunately rejected the method without even giving it a trial, for the reason that the dates ISS I. and $\mathrm{IS}_{7} \mathrm{~S}_{3}$, corresponding to the values $\mathrm{I} 3 \mathrm{I}$ and $\mathrm{r} 33$ of $s$; were very imperfectly determined. It was clear that the additional point thus secured would deserve very little weight. It was thought to diminish the irregularity of the line by combining these with previous and better determined dates. Mr. Pickering has not only used this method (which properly used is capable of yielding good results), but he has given equal weights to the values of $\frac{d s}{d T}$ for all the dates in the table. This is the fatal defect which entirely vitiates the conclusion reached by him. A reference to Fig. I of this paper will show that for the earlier dates from $\mathrm{I} S 54 \cdot{ }_{0}$ down to $\mathrm{I} S 72 .{ }_{6}$ the graphically determined dates differ from the real dates when the record was actually lowered by from one to two years.

It will also be seen that the dates ${ }_{1} S_{7} S_{._{3}}$ and ${ }_{I} S S_{1_{0}}$ are subject to errors which may be as great as two years. After having made a preliminary examination, these dates might indeed have been "adjusted" so as to make them agree better with the others, but they now stand exactly as they did when first made and before any other work had been done. It is clear that the most weight should be given to the earlier dates. I have therefore plotted the new values of $\frac{d s}{d T}$ with the values of $s$, and have drawn the line representing the values so as to give most weight to the best determined values. The equation of this line is

$$
\frac{d s}{d T}=-\mathrm{I}_{24}+0.0127 \mathrm{~s} . \quad-\quad-\quad-
$$

From this equation the values of $\frac{d s}{d T}$ were calculated as given in the fourth column of the last table. The fifth column, headed $e$, gives the time in years by which the corresponding time in- 
tervals $d T$ must be increased in order to bring Mr. Pickering's values of $\frac{d s}{d T}$ into accordance with the values calculated from the above equation. In this case the intervals are supposed to be separately adjusted. If the later dates were simultaneously ad. justed or changed by intervals ranging from two-tenths to threefourths of a year, the values of $\frac{d s}{d T}$, which Mr. Pickering prefers to use, would agree exactly with the values calculated from the last equation. Now it is perfectly clear that these later dates, and particularly the last two, are subject to just such errors as this.

Whatever these values of $\frac{d s}{d T}$ may be said to prove, they certainly do not prove that my results as before published are absurd, and they do not indicate a limiting speed of -25 seconds. If $\frac{d s}{d T}=0$, the limiting speed of the horse is found to be $9 \delta$ seconds.

I desire to express my thanks to Mr. Pickering for his criticisms and suggestions, as he has corrected a tendency which I had begun to feel, to attach too much importance to the numerical result reached; but I maintain that his method, correctly applied, gives in general, substantially, the same result as my own. It is not necessary to assert that this result is really correct if any person feels inclined to doubt it, because at present it is not possible to demonstrate it more fully than has been done in the present paper. I only insist that it is not wholly unwarranted by the facts which we now know.

Nost horsemen seem to think that the limiting speed of the trotting-horse will be sometwhere near a mile in rzo seconds. If this were true, the differential equation could hardly be a linear one. The equation

$$
\frac{d s}{d T}=k \sqrt{ } s \overline{-L} \quad-\quad-\quad-\quad-\quad-
$$

where $k$ is a constant and $L$ is the limiting speed, would however be in harmony with this view. But this equation gives on integration an equation of the form

$$
\sqrt{s-L}=C-A T \quad-\quad-\quad-\quad-
$$

According to this equation the horse would absolutely reach the limiting speed $L$ in a finite time, $\frac{C}{A}$. Practically this may be true, as is in fact shown by my own equation (4), so that some such equation might really represent the results suffi : $:$ eritly rear 
for all practical purposes. But the relation is not a rational one, since it cannot be supposed that the horse will really attain his limiting speed in a finite time. After he had come within a thousandth of a second, it would take a great interval of time to compass the next millionth of a second. Furthermore, this equation could not hold for values of $T$ greater than $\frac{C}{A}$, as the value of $s$ would then begin to increase according to equation ( $S$ ). I therefore claim that equation (4), in all probability, represents the relation between the values of $s$ and $T$, and that the constants in the equation will be determined with greater and greater precision as the data becomes more and more complete.

\section{Magnetic Survey of Missouri. Fifth Annual Report.}

By Francis E. Nipher.

During the summer of $1 \$ 82$ the survey was continued under the same auspices as in the previous year. The friend who furnished the entire means for conducting the work enlarged upon his former bounty, and furnished the party with two fine springwagons designed with reference to the needs of camp life, and provided with all needed conveniences. Two paid assislants, Messrs. Joseph Cunningham and Albert Meyer, were also sent with the expedition, and Mr. Frank Ringling of the Sophomore class accompanied the expedition as volunteer assistant, paying his own expenses.

The work of the summer was interfered with in a serious manner by the sickness of the horses, and more particularly by the horrible condition of the roads, due to heavy and long-continued rains. During the entire summer we were compelled to improve roads and fords, and to build bridges, and this frequently took up a quarter of our time during an entire week. This made it impossible to make complete observations after Aug. 7 th, as we were obliged to travel every day in order to reach St. Louis within the time which could be devoted to the survey. After the above date, therefore, only declination observations were made, the magnetic meridian being determined from the morning elonga- 
tion as explained in the previous report.* The true meridian was in nearly all cases obtained by pole-star observations, but the great number of cloudy nights made it necessary in some cases to observe at other times than at elongation.

A description of the stations where observations were made is here given, the numbers being continued from the previous report.

Station ioi - Kirkwood, St. Louis Co. Lat. $38^{\circ} 36^{\prime}$; lon. $90^{\circ} 24^{\prime}$. In the orchard of $\mathrm{H}$. W. Leffingwell, 128 feet from the street fence on the south, and ${ }^{5} \mathrm{O}$ feet from that on the east. Polaris observation on elongation.

Station roz-Gray's Summit, Franklin Co. Lat. $38^{\circ} 29^{\prime}$; lon. $40^{\circ}$ $49^{\prime}$. On the Union road about half a mile to the S. W. from the railroad crossing, and in the second depression, $20 \mathrm{ft}$. from the road, and on the N.W. side. Polaris obs. on elongation.

Station 103-Newport, Franklin Co. Lat. $38^{\circ} 36^{\prime}$; lon. $91^{\circ} 06^{\prime}$. In the "old town" on the summit of a small ridge, $128 \mathrm{ft}$. N.W. from the N.W. corner of the church. A large elm to the N.W. across the small watercourse is said to be at, or very near, the N.W. corner of the S.E. qr. of sec. II, tp. 44, r. 2 W. Polaris obs. on elongation.

Station ro4-On the farm of August Goebel, near Newport. Lat. $38^{\circ}$ $34^{\prime}$; lon. $91^{\circ} 06^{\prime}$. The station was within a few feet of the middle point of the line dividing the $\mathrm{E}$. half of the S. W. qr. from the W. half of the S.E. qr. of sec. 15 , tp. 44, r. 2 W. Polaris obs. on elongation. In this immediate vicinity Dr. Goebel, grandfather of the present owner of the farm, had established two magnetic stations, where he made extended and careful observations. The original records of this work were given to the U.S. Coast Survey some years since by his son, Mr. Gert Goebel.

The earlier station of Goebel is found by going from our station in a line bearing S. $93^{\circ} 34^{\prime} \cdot{ }_{5}$, E. $255 \mathrm{ft}$. and thence N. $83 \mathrm{ft}$. This station of Goebel's is $61 \mathrm{ft}$. W. and $44 \mathrm{ft}$. N. of the S.W. corner of the house of August Goebel. The observations here were made in the year 1839. The declination was $9^{\circ} 2 \mathrm{I}^{\prime} \mathrm{E}$.

The other station of Dr. Goebel was occupied in the year I849. It is at the $\mathrm{S}$. window of a now abandoned stone house, the N.W. corner of which is $158 \mathrm{ft}$. W. and $193 \mathrm{ft}$. S. of our station. The house is a one-story structure, having the dimensions of $\mathrm{N}$. and $\mathrm{S}$. sides $\mathrm{i} 8 \mathrm{ft}$., and $\mathrm{E}$. and $\mathrm{W}$. sides $24 \mathrm{ft}$. The window where his observations were made is in the middle of the $\mathrm{S}$. side. His value for declination in 1849 was $9^{\circ} 05^{\prime} \mathrm{E}$. Our value determined June $22 \mathrm{~d}$, 1882 , was $7^{\circ} 36^{\prime}$.

Station ro5-In Franklin Co. Lat. $38^{\circ} 44^{\prime}$; lon. $91^{\circ} 20^{\prime}$. The station is on timber land of Elijah Ruck, $315 \mathrm{ft}$. N. and $\mathrm{I}_{5} \mathrm{E}$. of the well in 
front of the house of John Bedts, and about $325 \mathrm{ft}$. from the line between Franklin and Gasconade counties. The station was said to be in sec. Io, tp. 45, r. 4 W. Polaris obs. on elongation.

Station ro6-In Gasconade Co. Lat. $3 S^{\circ} 37^{\prime}$; lon. $91^{\circ} 29^{\prime}$. On the land belonging to Fred. Bruhns, a quarter of a mile N. and $260 \mathrm{ft}$. E. of the S.IV. corner of sec. I9, tp. $45, \mathrm{r} .5 \mathrm{~W}$. The station is on the bank of First creek. The value for declination here was very much smaller than at surrounding stations, but no error could be detected in our work. Whether the discrepancy was due to some minute local effect or not, we could not remain to determine, as sickness at home made it desirable to reach the telegraph as soon as possible. This is the only case of the kind so far reached, excepting in the Ison Mountain region. Two Polaris observations were made on elongation.

Station io7-In Osage Co. Lat. $3^{\circ}{ }^{\circ} S^{\prime}$; lon. $9 \mathrm{l}^{\circ} 4^{\mathrm{I}^{\prime}}$. On the land of Fritz Kaldeweiher, near the centre of the S.E. qr. of the S.WV. qr. of sec. 9 , tp. 43. r. 7 W. The station was $50 \mathrm{ft}$. N. and $33 \mathrm{ft}$. W. of the N.W. corner of Kaldeweiher's house. Polaris obs, on elongation.

Station $108-L i m$, Osage Co. Lat. $38^{\circ}{ }_{2} S^{\prime}$; lon. $91^{\circ} 50^{\prime}$. Station on I'Ours creek* on the Jefferson city road, about half a mile fiom town. The S.E. corner of James N. Clark's yard is $72 \mathrm{ft}$. W. and $68 \mathrm{ft}$. S. of the station. Polaris obs. on elongation.

Station 109-In Callaway Co. Lat. $3 S^{\circ} 43^{\prime} ;$ lon. $92^{\circ}$ oI'. On Little Auxvasse creek at the crossing of the New Bloomfield and Fulton road, about 40 rods S.W. of the N.E. corner of sec. $2 S$, tp. 46 , r. Io IV. A spring across the creek and just at the ford lies N. $65^{\circ}$ E. $165 \mathrm{ft}$. Polaris obs. on elongation.

Station in-Near Stephens' Store, Boone Co. Lat. $3 S^{c} 5^{S^{\prime}} ;$ lon. $92^{\circ}$ 05'. About one half mile $S$. of the village. The old Fulton road is $\mathbf{2} 2 \mathrm{ft}$. W. and the bank of Cedar creek is $105 \mathrm{ft}$. N. Polaris obs. on elongation.

Station in - Centralia, Boone Co. Lat. $39^{\circ}$ I $^{\prime}$; lon. $92^{\circ}$ 05'. The station was on a vacant lot $4 \mathrm{~S} \mathrm{tt}$. W. of the centre of the street leading directly S. to the depot and crossing of the Chicago $\&$ Alton R.R., which is about a square and $\mathrm{a}$ half distant. The centre of the street to the $\mathrm{N}$. is distant $77 \mathrm{ft}$. and leads E. to a flouring mill, the smokestack of which bears S. $90^{\circ} 44^{\circ}, 1$ E. Polaris obs. on elongation.

Station itz-In Monroe Co. Lat. $39^{\circ} 24^{\prime}$; lon. $92^{\circ}$ Io $^{\prime}$. On the S. bank of the Long Branch of Salt river. The station was $150 \mathrm{ft}$. from the creek and midway between the road and the W. fence. 'The station is on the W. line of sec. 20 , tp. 53. r. I I. W., and abont r $20 \mathrm{yds}$. from the middle of this line. Polaris obs. on elongation. - Some years since the county surveyor of Audrain Co. called my attention to this region as showing

* Named after the first (French) settler on its banks. A postoflice near the stream was afterwards named "Loose Creek" P. O. by some poor speller, and this oflicial name has since been applied to the stream, which appears on some maps as "Lonse Creek." 
marked local effects. The region is level prairie, and long N. and S. lines run by compass are greatly and uniformly curved, showing an abnormally great easterly declination. This station and station No. I44, about I2 miles E., show an area of abnormal easterly declination. The disturbed region extends over an area of many miles.

Station 1 13-Moberly, Randolph Co. Lat. $39^{\circ} 26^{\prime}$; lon. $92^{\circ} 26^{\prime}$. On the fair grounds $1 S_{4} \mathrm{ft}$. E. and $2 S_{9} \mathrm{ft}$. S. of the W. entrance. Polaris obs. on elongation.

Station $114-$ Macon, Macon Co. Lat. $39^{\circ} 46^{\prime}$; lon. $92^{\circ} 30^{\prime}$. In the stock-yard of O.S. Bearce, directly in front of his barn and $20 \mathrm{ft}$. from the Vine-st. fence. The city school-house is one square $\mathrm{W}$. and one square $\mathrm{N}$. Polaris obs, on elongation.

Station 115-In Macon Co. Lat. $39^{\circ} 4^{\prime} S^{\prime}$; lon. $92^{\circ} 37^{\prime}$. On the farm of Isaiah Lewis, which is the N. half of N.E. qr. of sec. $2 \mathrm{I}$, tp. 5 S, r. I5 W. The station was in front of the house, and midway between the road and the yard fence. Polaris obs. on elongation.

Station i $6-$ Near Mercyville, Macon Co. Lat. $39^{\circ} 57^{\prime}$; lon. $92^{\circ} 42^{\prime}$. About half a mile N. of town. A corner-stone in the road, a quarter of a mile S. of the middle point of the N. line of sec. 35 , tp. 60, r. $16 \mathrm{~W}$., bears S. $3^{\circ} 23^{\prime} \mathrm{W}: 589 \mathrm{ft}$. Polaris obs. on elongation.

Station in 7 In Linn Co. Lat. $39^{\circ} 54^{\prime}$; lon. $93^{\circ} \mathrm{o} 7^{\prime}$, In the bottom of the west branch of Yellow creek. The station is within a few feet of the corner, a quarter of a mile due E. of the middle point of the E. line of sec. 22 , tp. 59 , r. I9 W. A large white-oak tree stands $20 \mathrm{ft}$. W., and the east end of the bridge is $26 \mathrm{It}$. distant. The mark used was an iron rod on the bridge, II ft. west of the centre of the bridge. Polaris obs. on elongation.

Station $18-$ Linnceus, Linn Co. Lat. $39^{\circ} 5^{\text {I }^{\prime}}$; lon. $93^{\circ} 13^{\prime}$. On a vacant lot on the summit of the hill E. of the Burlington \& South-western R.R. depot. The S. line of the yard of Chas. B. Purdin lies I66 ft. N., the N. line of the farm of Joel Wilkinson lies $166 \mathrm{ft}$. S., while the field to the E. across the road is $237 \mathrm{ft}$. distant. The court-house spire was used as a mark. Two polaris observations on elongation.

Station 119-Near Laclede, Linn Co. Lat. $39^{\circ} 47^{\prime}$; lon. $93^{\circ} 17^{\prime}$. Station one mile W. of town, on the E. side of Muddy creek; about $35^{\circ} \mathrm{ft}$. E. of the bridge and $20 \mathrm{ft}$. N. of the road centre. The station is said to be on the S E. qr. of sec. 36 , tp. 5 S, r. 2 I W. Polaris obs. on elongation.

Station 120-In Livingston Co. Lat. $39^{\circ} 38^{\prime}$; lon. $93^{\circ} 45^{\prime}$. On the farm of Wm. E. Wolfort, in the N.E. qr. of the S.E. qr. of sec. 33, tp. 56, r. $25 \mathrm{~W}$. The station was in the cattle-yard $25 \mathrm{ft}$. from the road fence to the east (the road being on the section line), and $40 \mathrm{ft}$. N. of the door-yard fence. The middle line of the section is in the E. and W. road perhaps, $100 \mathrm{ft}$. N. Polaris obs. on elongation.

Station $121-$ Kingston, Caldwell Co. Lat. $39^{\circ} 4^{1^{\prime}}$; lon. $94^{\circ} 04^{\prime}$. The station will be found by going from the S.IV. corner of the court-house 
square W. $1646 \mathrm{ft}$, and $\mathrm{S}$. from the middle of the road $62 \mathrm{ft}$. It lies $9 \mathrm{I} \mathrm{ft}$. E. of the summit of the small ridge. Polaris obs. on elongation.

Station 122 - In Caldzull. Co. 'Lat. $39^{\circ} 39^{\prime} ;$ lon. $94^{\circ}$ II'. On land of Christian Sinitt, $50 \mathrm{ft}$. W. and $212 \mathrm{ft}$. N. of the middle of sec. $29, \mathrm{tp} .5^{6}$, r. $29 \mathrm{~W}$. Polaris obs on elongation.

Station r23-May'sville, DeKalb Co. Lat. $39^{\circ} 43^{\prime}$; lon. $94^{\circ} 24^{\prime}$. On the grounds of the public school building, $65 \mathrm{ft}$. W. and $2 \mathrm{I} \mathrm{ft}$. N. of the N.W. corner of the building. Polaris obs. on elongation.

Station r $24-$ In DeKalb Co. Lat. $40^{\circ}$ o1'; lon. $94^{\circ} 23^{\prime}$. On land of Harvey Johnson, $50 \mathrm{ft}$. S. and $206 \mathrm{ft}$. E. of the middle of the N. line of sec. I 4 , tp. 6o, r. 3I W. Polaris obs. on elongation.

Station 125-Albany, Gentry Co. Lat. $40^{\circ}$ 15 $^{\prime}$; lon. $94^{\circ} 21^{\prime}$. The station is $979 \mathrm{ft}$. E. of the N.E. corner of the court-house square and $8 \mathrm{ft}$. $\mathrm{S}$., these measurements being along the streets. The station is II $\mathrm{ft}$. W. and $8 \mathrm{ft}$. S. of the N.E. corner of lot 2 , block 5 , of Hundley's second addition. Polaris obs. on elongation.

Station $126-$ In Gentry Co. Lat. $40^{\circ}$ I6 $^{\prime}$; lon. $94^{\circ} 7^{\prime}$. The fence E. on the E. edge of sec. 15, tp. 63, r. $30 \mathrm{~W}$., is $107 \mathrm{ft}$. distant. The fence $\mathrm{S}$, which is the $\mathrm{S}$. line of the N. half of the N.E. qr. of the section, is 193 ft. distant. There appears to be a double corner here. The evening-mark reading was missed, but the station was on raw prairie and the instrument was certainly not disturbed between the star observation and the morning mark reading. Polaris was observed on elongation.

Station r27-Bethany, Harrison Co. Lat. $40^{\circ}$ I $^{\prime}$; lon. $94^{\circ} \mathrm{o} 3^{\prime}$. On a vacant lot of Mrs. R. J. Turner, $365 \mathrm{ft}$. E. of the N.E. corner of her house. The station is about $320 \mathrm{ft}$. S. of the N. line and $346 \mathrm{ft}$. W. of the E. line of sec. 15, tp. 63 , r. $28 \mathrm{~W}$. The line fence of T, B. Shearer's yard is $101 \mathrm{ft}$. E. Polaris obs, on elongation.

Station 128-Farm of Fohn Honan, in Harrison Co. Lat. $40^{\circ}$ o S' $^{\prime}$; lon. $93^{\circ} 56^{\prime}$. The station was in the meadow, $133 \mathrm{ft}$. S. and $289 \mathrm{ft}$. W. of the N.E. corner of sec. 36, tp. 62, r. 27 W. Meridian determined by equal altitudes of the sun. Small cumulus clouds cut off five observations out of a series of seven. 'The two differed 1 '.

Station I29-In Daviess Co. Lat. $40^{\circ} 04^{\prime} ;$ lon. $93^{\circ} 53^{\prime}$. The station was in the road ahout midway between the track and the S. fence, and 334 ft. E. of the N.W. corner of the S. half of the S.IV. qr. of sec. 28, tp. 6I, r. $26 \mathrm{~W}$. By reason of a very heavy rain which came up while the camp was being made it was impossible to get an evening-mark reading. The rain lasted until 9:15 P.M., and then a small patch of sky cleared around polaris for about half an hour, and a pole-star observation was made.?

When the star-observation was inade the whole hillside was covered with a sheet of water three to four inches inches in depth, which filled the trenches around the tent and ran through the tent in a torrent. The ground was however firm, and the tripod was as usual mounted firmly on 
large stakes driven eight to ten inches into the ground. The observation was therefore deemed entirely satisfactory. At the next station on the next night an observation was made the same interval before elongation, and the difference between the azimuth of the star and that of elongation agreed within a quarter of a minute with that at Station 129.

Station I3o-Trenton, Grundy Co. Lat. $40^{\circ}$ \%3'; lon. $93^{\circ} 39^{\prime}$. Station in a grove of Dr. Harris, in the east part of town. The station is found by starting at the front (S.) fence of the door-yard and measuring S. along the centre of the road in $2 \mathrm{ft}$., thence E. $4 \mathrm{I} 5 \mathrm{ft}$. Polaris obs. on elongation.

Station r3 3 - In Grundy Co. Lat. $40^{\circ} 1^{\prime}$; lon. $93^{\circ} 38^{\prime}$. Station in the road about midway between the track and the E. fence, and $150 \mathrm{ft}$. S.W. from the front gate of the farm of Faust Amick. The station is near the S.E. corner of the N.E. qr. of N.E. qr. of sec. 34 , tp. 63, r. 24 W. Polaris was observed before elongation, and its azimuth calculated as before described.

Station 132-Princeton, Mercer Co. Lat. $40^{\circ} 24^{\prime}$; lon. $93^{\circ} 39^{\prime}$. The station is at the base of the bluff, $395 \mathrm{ft}$. W. of the W. side of Lincoln st. and $563 \mathrm{ft}$. N. of the centre of Hickland st. Polaris observations were made at 9 and $12 \mathrm{~h} .30 \mathrm{~m}$.

Station 133 -In Putnam Co. Lat. $40^{\circ} 27^{\prime}$; lon. $93^{\circ} 21^{\prime}$. On land of Joseph Williams, about a quarter of a mile W. of the centre of sec. 7 , tp. 65, r. 2 I W. The station was about $20 \mathrm{ft}$. N. of the centre of the road, and $250 \mathrm{ft}$. E. of the front gate of the house of Crede Yocum. Polaris obs. on elongation.

Station $134-$ In Putnam Co. Lat. $40^{\circ} 27^{\prime}$; lon. $93^{\circ} 2 \mathrm{r}^{\prime}$. Station in a lane near the house of Joseph Ward, in sec. 12, tp. 65, r. 20 W. The middle stone of the $\mathrm{S}$. line of the section is $\mathrm{I}_{9} \mathrm{ft}$. S. of the station. Pola. ris observed at I I O'clock P.M.

Station i35-Unionville, Putnam Co. Lat. $40^{\circ} 29^{\prime}$; lon. $93^{\circ} \mathrm{o} 3^{\prime}$. The station is on an open square $562 \mathrm{ft}$. N.W. of the W. corner of the courthouse square and $150 \mathrm{ft}$. N.W. of the same. These measurements are made along the streets which lie diagonally in reference to the points of the compass. The sky was again cloudy at elongation, and polaris was observed at $9 \mathrm{~h}$. I I m. and Io h. $46 \mathrm{~m}$.

Station $136-I n$ Sullivan Co. Lat. $40^{\circ}$ I9'; lon. $93^{\circ}$ o $7^{\prime}$. On the farm of Nathan Bankes, on the N.E. qr. of the S.E. qr. of sec. 28, tp. 64 , r. Ig W. The station is in the meadow $26 \mathrm{ft}$. S. of the S.W. corner of Bankes' house and $249 \mathrm{ft}$. W. of the middle of the road. Polaris obs. on elongation.

Station $137-$ Milan, Sullivan Co. Lat. $40^{\circ} \mathrm{I}^{\prime}$; lon. $93^{\circ} \mathrm{II}^{\prime}$. On the common, $160 \mathrm{ft}$. S. of the S.W. corner of the public school building. Polaris obs. on elongation. 
Station ${ }^{8} 3^{8-S t i c k l e r v i l l e, ~ S u l l i v a n ~ C o . ~ L a t . ~} 40^{\circ} \mathrm{c} 9^{\prime}$; lon. $92^{\circ} 5^{8 \prime}$. Near the S.IV. corner of the N.W. qr. of sec. 22, tp. 62, r. 18 W. The N. W. corner of the church bears S. $3 \mathrm{I}^{\circ}{ }_{1} 5^{\prime} \mathrm{W}$., and is distant $731 \mathrm{ft}$. The same corner of the church is $137 \mathrm{ft}$. S.E. of the qr. sec. corner before mentioned. Polaris obs, not at elongation.

Station 139-Kirksville, Adair Co. Lat. $40^{\circ} 12^{\prime}$; lon. $92^{\circ} 37^{\prime}$. In a vacant lot, owned by Dr. Hurley, on the S.W. corner of Fifth and Fillmore sts. $76 \mathrm{ft}$. from the centre of the latter and $83 \mathrm{ft}$. from the centre of the former street. 'The left side of the tower of the State Normal school building bears S. $69^{\circ} \mathrm{O}^{\prime} \cdot{ }_{5} \mathrm{E}$. Polaris obs, on elongation.

Station i $40-$ La Plata, Macon Co. Lat. $40^{\circ} \mathrm{oO}^{\prime}$; lon. $92^{\circ} 34^{\prime}$. Station in the street about midway between the track and the $\mathbf{N}$. fence. The middle, E. and W. line of sec. 7 , tp. 60, r. 14 W., is $486 \mathrm{ft}$. N., and the E. line of the section is $637 \mathrm{ft}$. E. These distances were measured along the streets. The house of B. F. Bragg is on the S. side of the street, a little E. of the station. Polaris obs. on elongation.

Station 141 - In Macon Co. Lat. $39^{\circ} 53^{\prime}$; lon. $92^{\circ} 22^{\prime}$. Station on Bear creek bottom, $304 \mathrm{ft}$. N. and $832 \mathrm{ft}$. E. of the middle of the S line of sec. 23 , tp. 59, r. 13 W., near Harris's farm. Some error was made in reading the verniers in the star observation. It is conjectured that the altitude was read too high by $10^{\prime}$. This conjecture is baesd on observations at the next station. made at elongation and an equal interval after elongation, allowance being made for the change in latitude.

In the magnetic determination on the morning of the 12 th marked disturbances of the needle were observed. The declination diminished $9^{\prime}$ between 6 and 7 o'clock.

Station $142-$ In Shelby Co., 3 miles S. of Shelbyville. Lat. $39^{\circ} 44^{\prime}$; lon. $92^{\circ} \mathrm{O}^{\prime}$. The middle stone on the N. line of sec. 5, tp. 57, r. 10 W., bears N. $4^{\circ}, 50^{\prime}$ E., and is distant 9 Io ft. Polaris obs. on elongation.

Station 143-In Monroe Co., on the farm of Henry Winkler, $40 \mathrm{ft}$. E. of the centre of the road, and $669 \mathrm{ft}$. N. of the S. line of sec. $35, \mathrm{tp} .56, \mathrm{r}$. so W. The road, which runs $\mathrm{N}$. and S., divides the S.W. qr. of the section in halves. Polaris obs. on elongation.

Station 144- In Monroe Co. Lat. $39^{\circ} 22^{\prime}$; lon. $91^{\circ} 59^{\prime}$. On the summit of the S. bluff of Long Branch of Salt river, W. of the road, on land of B. F. Dowell. The land is in sec. 30 , tp. 53, r. 9 W. The station was about $75 \mathrm{ft}$. W. of the road, which is on the E. line of the section. The Baptist church across the road is about $50 \mathrm{ft}$. farther $\mathrm{S}$. than the station. These measurements were forgotten, and the distances were estimated the same day after having left the locality. Polaris observation on elongation.

Station 145-Montgomery City. Lat. $39^{\circ} \mathrm{oo}^{\prime}$; lon. $9 \mathrm{I}^{\circ} 3 \mathrm{O}^{\prime}$. The station is $105 \mathrm{ft}$. W. of the middle (N. and S.) line of the S.E. qr. of sec. $3^{2}$, tp. 49 , r. 5 W., and a perpendicular laid off to the track of the Wabash- 
Pacific R.R. track measures $200 \mathrm{ft}$. Passing freight trains caused the needle to swing through $2^{\prime}$. Polaris obs. on elongation.

Station 146-Warrenton, Warren Co. Lat. $38^{\circ} 46^{\prime}$; lon. $91^{\circ} \mathrm{os}^{\prime}$. The station is at the W. end of town, about a mile from the station of $188 \mathrm{I}$. Starting at the creek bridge $\mathrm{W}$. of the court-house, the station will be found by going along the road westwardly $705 \mathrm{ft}$., thence southwardly at right angles to the road a distance of $45 \mathrm{ft}$. The court-house spire bears S. $79^{\circ}$ 14. ' ' E. Polaris obs. on elongation.

Station 147-In St. Charles Co. Lat. $38^{\circ} 43^{\prime}$; lon. $90^{\circ} 40^{\prime}$. The station is in the Booneslick road about midway between the track and the $\mathrm{S}$. fence, and almost due south of O'Fallon.* The O'Fallon road is $250 \mathrm{ft}$. E. The house of $\mathrm{I}$. Heald lies a few rods to the W. Polaris observation on elongation.

Sration $148-I$ in St. Lou is Co. Lat. $38^{\circ} 4^{I^{\prime}}$; lon. $90^{\circ} 2 \mathrm{I}^{\prime}$. On the St. Charles rock road. The station was in a gap in the fence opposite the grounds of J. B. Lucas. From the station to the centre of the road the distance is $30 \mathrm{ft}$. From thence along the road to a point opposite the gate is $165 \mathrm{ft}$., while the distance in the opposite direction to a point opposite the S.E. corner of the Lucas grounds is $15^{8} \mathrm{ft}$. Polaris observation on elongation.

Station i 49-Near Atalissa, Muscatine Co., Iowa, on the farm of Mrs. Grace Aikins, on the N.E. qr. of the N.W. qr. of sec. 3, tp. 78, r. 3 W. The station is on the front path, exactly between the front gate and the house. This station is a mile west of station 33 in the report of r880. $\dagger$ Polaris obs.

Station 34-This station was occupied in IS80, and is described in the report for that year. Polaris obs. on elongation.

At six of the stations of the summer deflection determinations were made with the University magnetometer, with magnet $\mathrm{C}_{6}$ deflecting and $\mathrm{C}_{17}$ deflected, and these observations have been used in determining the value of $P$, and in calculating the magnetic moment of $\mathrm{C}_{6}$ for the summer. The latter was sensibly constant, the observed difference between the extreme value observed and the mean of all being about $0.000 s$ of the average moment. In the reduction of the work the magnetic moment was therefore assumed to be constant. The calculations for $P$ and for the magnetic moment are given in the adjoining tables.

\footnotetext{
* In the report for iSso the Ion. of O'Fallon should be $99^{2} 40^{\prime}$.

t Station 33 is on the N.E. qr. of the N.W. qr. of sec. 2, and not N.E. qr. of N.E. qr. as was given in the report of $\mathrm{I} 3 \mathrm{So}$.
} 


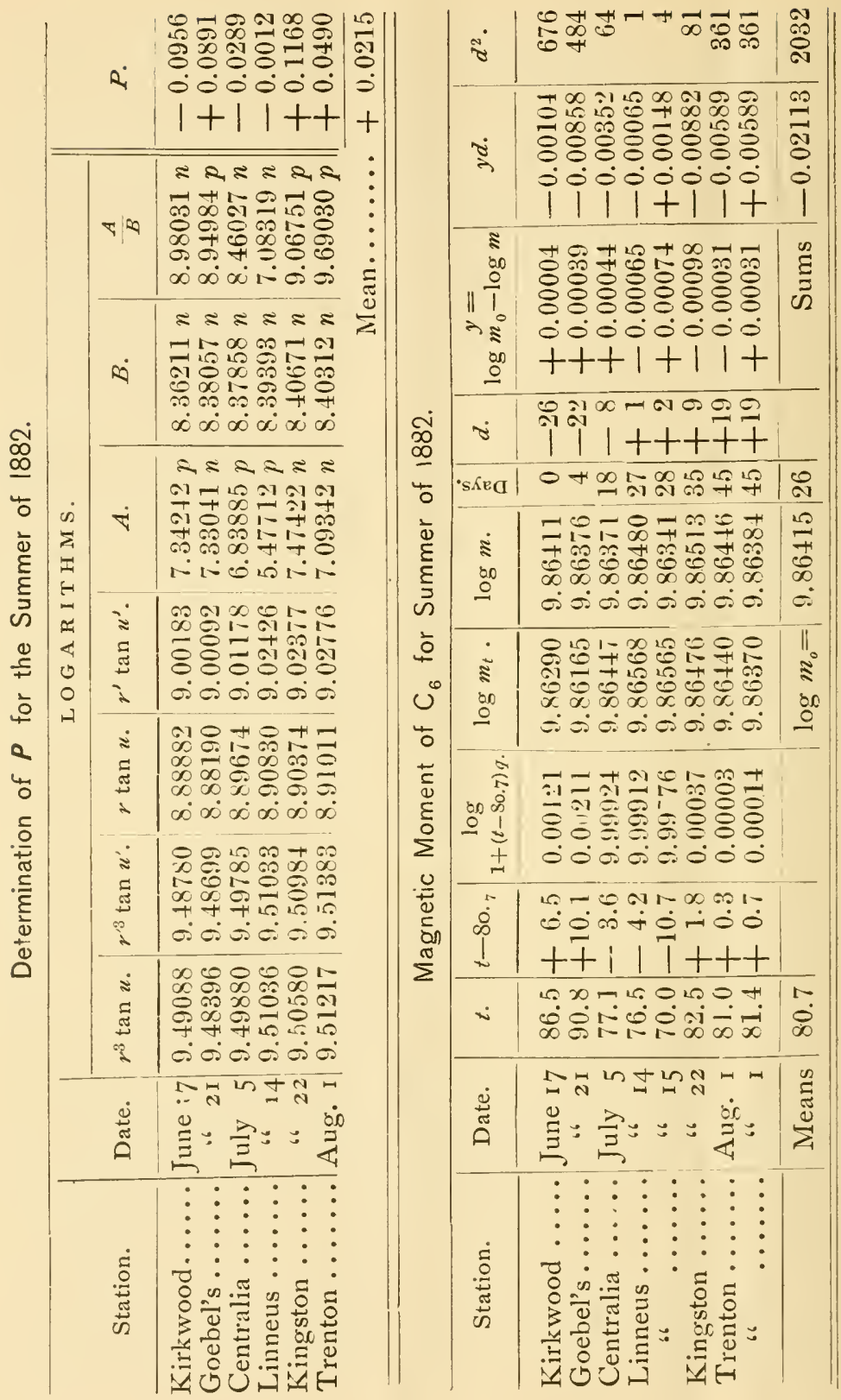


NIPHER- $5^{\text {th }}$ ANN. REP. MAGNETIC SURVEY OF MISSOURI. 525

The value of $P$, as determined in the first of the tables, gives for the value of $\mathrm{I}-\frac{P}{r^{2}}$ the values

$$
\begin{array}{cll}
1-\frac{P}{2^{2}} & =0.9946 & \log =9.99765 \\
1-\frac{P}{\left(I_{75}\right)^{2}}=0.9930 & \log =9.99695
\end{array}
$$

These values were used in the reduction of the deflection series made for the determination of the magnetic moment of magnet $\mathrm{C}_{6}$.

In the second table, the decrease $(a)$ in the value of $\log m$ is determined, and it is found that for the summer of 1882 , and at a temperature of $\mathrm{So}_{7}$, the value of $(a)$ is

$$
a=\frac{-0.02113}{2032}=-0.000,009 \text {, }
$$

or $\log m=9.86415+0_{000.009} d$,

where $d$ is estimated in days from July i 3 . The value of $\log m$ was therefore considered constant during the summer.

In all of the intensity determinations the time of vibration was determined by means of a Waltham watch belonging to $\mathrm{Mr}$. Ringling. This watch had been cleaned just before leaving St. Louis, but it had not been rated. The error of the watch was determined, at intervals during the summer, by comparison with clock-beats from the observatory of Washington University, transmitted daily to the telegraph lines of various railways in the State. Its rate during the summer was a loss of 20 seconds per day, fluctuating however between 17 and 22 seconds. The correction on the time of vibration was $+0_{0016}$ second at all the stations, the time of vibration at the stations not varying sufficiently to change the value of this correction. The effect of neglecting this correction altogether would be equivalent to the effect of an error of half a degree in temperature.

The intensity determinations were all made with magnet $\mathrm{C}_{6}$ in the University declinometer, $\mathrm{C}_{17}$ being used as a deflected magnet. The moment of inertia of $\mathrm{C}_{6}$ was obtained from the table given in the $4^{\text {th report.* }}$ The values for $H$ are not corrected for 
the effect of magnetic brass-work of the magnetometer. This correction is given in the $4^{\text {th }}$ report, p. $47^{2}$.

The observations for inclination were not very satisfactory, as the axes of the needles were both bent during the early part of the summer.

The following observations for meridian, by equal altitudes of the sun, were made. The method of reduction has been explained in previous reports.

Sun Observations for Meridian.

\begin{tabular}{|c|c|c|c|c|c|c|c|c|c|}
\hline \multirow{2}{*}{\multicolumn{2}{|c|}{ Station. }} & \multirow[t]{2}{*}{ Date. } & \multicolumn{4}{|c|}{ Mean Time of Series. } & \multirow{2}{*}{\multicolumn{2}{|c|}{$t$. }} & \multirow[t]{2}{*}{$1 / 2\left(A+A^{\prime}\right)}$. \\
\hline & & & \multicolumn{2}{|c|}{ A. $\mathbf{M}$. } & \multicolumn{2}{|c|}{ P. M. } & & & \\
\hline \multicolumn{2}{|c|}{$\begin{array}{l}\text { Little Auxvasse Cr. } \\
\text { Honan's ......... }\end{array}$} & $\begin{array}{l}\text { June } 30 \\
\text { July } 30\end{array}$ & $\begin{array}{ll}\text { h. } & m \text {. } \\
9 & 36 \\
9 & 38\end{array}$ & $\begin{array}{l}s \\
58 \\
25\end{array}$ & $\begin{array}{ll}\text { h. } & m . \\
2 & 32 \\
2 & 23\end{array}$ & $\begin{array}{l}s . \\
40 \\
18\end{array}$ & $\begin{array}{l}\text { deg. } \\
37 \\
35\end{array}$ & $\begin{array}{l}m . \\
3 \cdot 7 \\
2 \cdot 5\end{array}$ & $\begin{array}{ll}\text { deg. } & m . \\
244 & 56.7 \\
176 & 38.5\end{array}$ \\
\hline $\log \Delta d^{\prime \prime}$ & $\begin{array}{c}\text { a. c. } \log \cos \\
\varphi .\end{array}$ & a.c. $\log \sin t$ & Cor. & \multicolumn{2}{|c|}{ South reads } & \multicolumn{2}{|c|}{ Mark reads } & \multicolumn{2}{|c|}{ Az, of Mark. } \\
\hline $\begin{array}{l}1.67112 \\
2.23805\end{array}$ & $\begin{array}{l}0.10777 \\
0.11660\end{array}$ & $\begin{array}{l}0.21992 \\
0.23384\end{array}$ & $\begin{array}{l}+0.8 \\
+3.2\end{array}$ & \multicolumn{2}{|c|}{$\begin{array}{l}244^{\circ} 57^{\prime} \cdot 5 \\
176 \\
1\end{array}$} & \multicolumn{2}{|c|}{$\begin{array}{l}180^{\circ} \mathrm{O} 2^{\prime} \cdot 0 \\
\mathrm{I} 8 \mathrm{C} \text { OI } \cdot 2\end{array}$} & \multicolumn{2}{|c|}{$\begin{array}{l}\text { S. } 64^{\circ} 55.5 \text { E. } \\
\text { S. } 3 \text { I } 9.5 \text { W. }\end{array}$} \\
\hline
\end{tabular}

Polaris Observations for Meridian.

\begin{tabular}{|c|c|c|c|c|}
\hline Station. & Lat. $\phi$. & Alt. h.* & Polar D. $\not$. & $\frac{1}{2} \mathrm{Sum}=\mathrm{S}$. \\
\hline Daviess Co., Sta. I29. & $40^{\circ} 04^{\prime}$ & $39^{\circ} 42^{\prime} \cdot 5$ & $I^{\circ} 19^{\prime} \cdot 3$ & $40^{\circ} 32 / .9$ \\
\hline Amick's...$\ldots \ldots \ldots$ & 40 I 3 & $3934 \cdot 0$ & $\therefore$ & $4033 \cdot I$ \\
\hline Princeton $\quad \ldots \ldots \ldots \ldots \ldots$ & $40 \quad 24$ & $\begin{array}{lll}39 & 46 & \cdot 0 \\
39 & 58 & .0\end{array}$ & “6 & 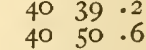 \\
\hline " $\quad \ldots \ldots \ldots \ldots$ & $r$ \& & $40 \quad 05 \cdot 0$ & " & $40 \quad 54 \cdot 2$ \\
\hline Ward's ........ & $40 \quad 27$ & $40 \quad 48 \cdot 0$ & “ & $41 \quad 17 \cdot 2$ \\
\hline ille... & $40 \quad 29$ & $40 \quad$ I2 $\cdot 5$ & “" & $4 \mathrm{I} 00 \cdot 4$ \\
\hline & & $40 \quad 45 \cdot 5$ & " & $41 \quad 16 \cdot 9$ \\
\hline$\underset{6 !}{\text { Sticklerville } \ldots \ldots \ldots} \cdot \ldots$ & $40 \quad 09$ & $3937 \cdot 0$ & “" & $40 \quad 32 \cdot 7$ \\
\hline arris's $\ldots \ldots \ldots \ldots \ldots \ldots$ & $39 \quad 53$ & $\begin{array}{lll}39 & 46 & \cdot 5 \\
40 & 25 & .0+\end{array}$ & "6 & $\begin{array}{lll}40 & 37 & \cdot 4 \\
40 & 48 & .6\end{array}$ \\
\hline & & & & $4040 \cdot 0$ \\
\hline
\end{tabular}

* The altitudes are all corrected for refraction.

$\dagger$ This altitude was recorded $40^{\circ} 36^{\prime} .0$. It should have been $40^{\circ} 26^{\prime}$, as was determined at the next station (Shelbyville) by an observation made the same time interval after elongation. 
NIPIIER $-5^{\text {th }}$ ANN. REP. MAGNETIC SURVEY OF MISSOURI. 527

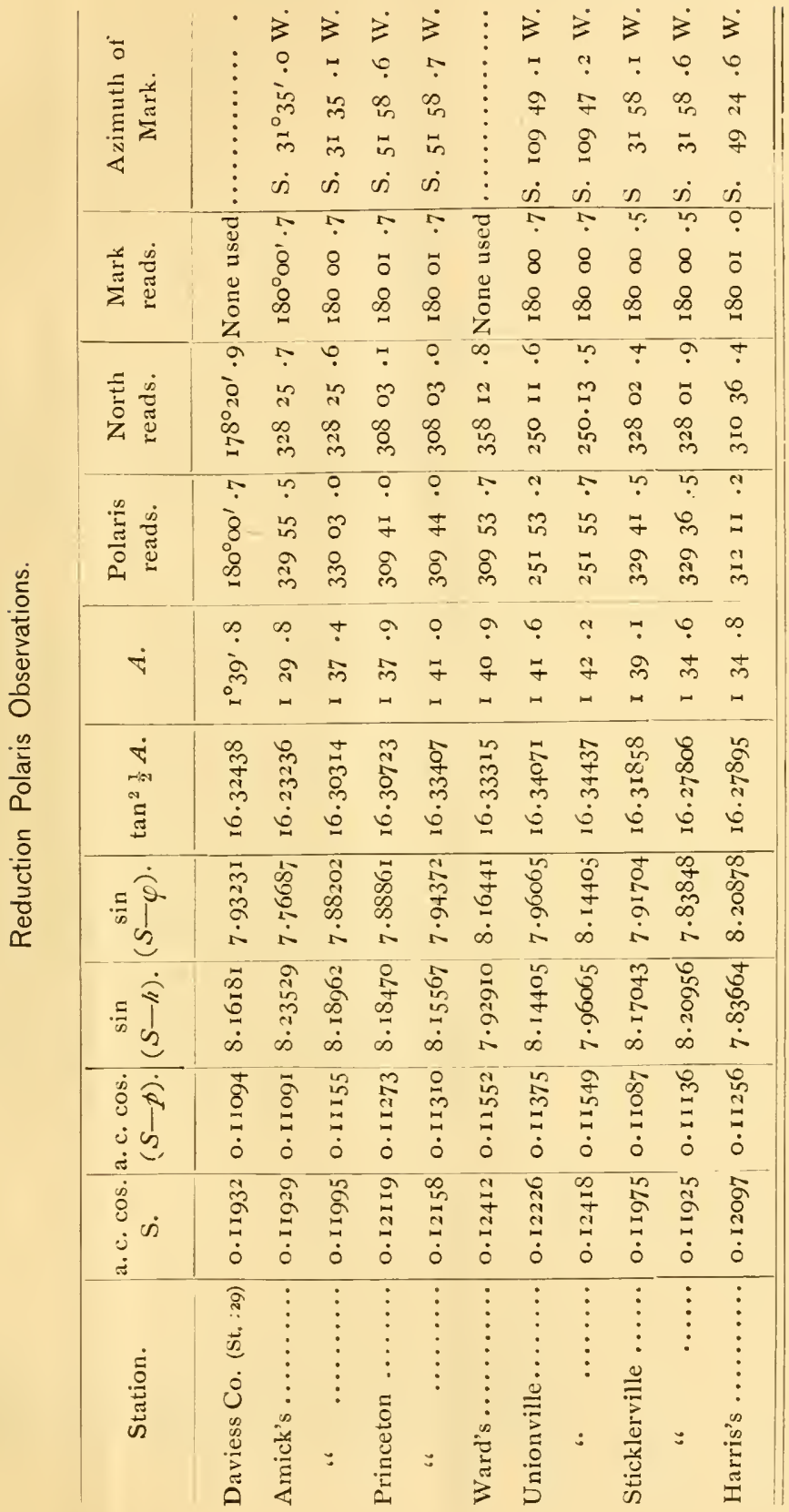




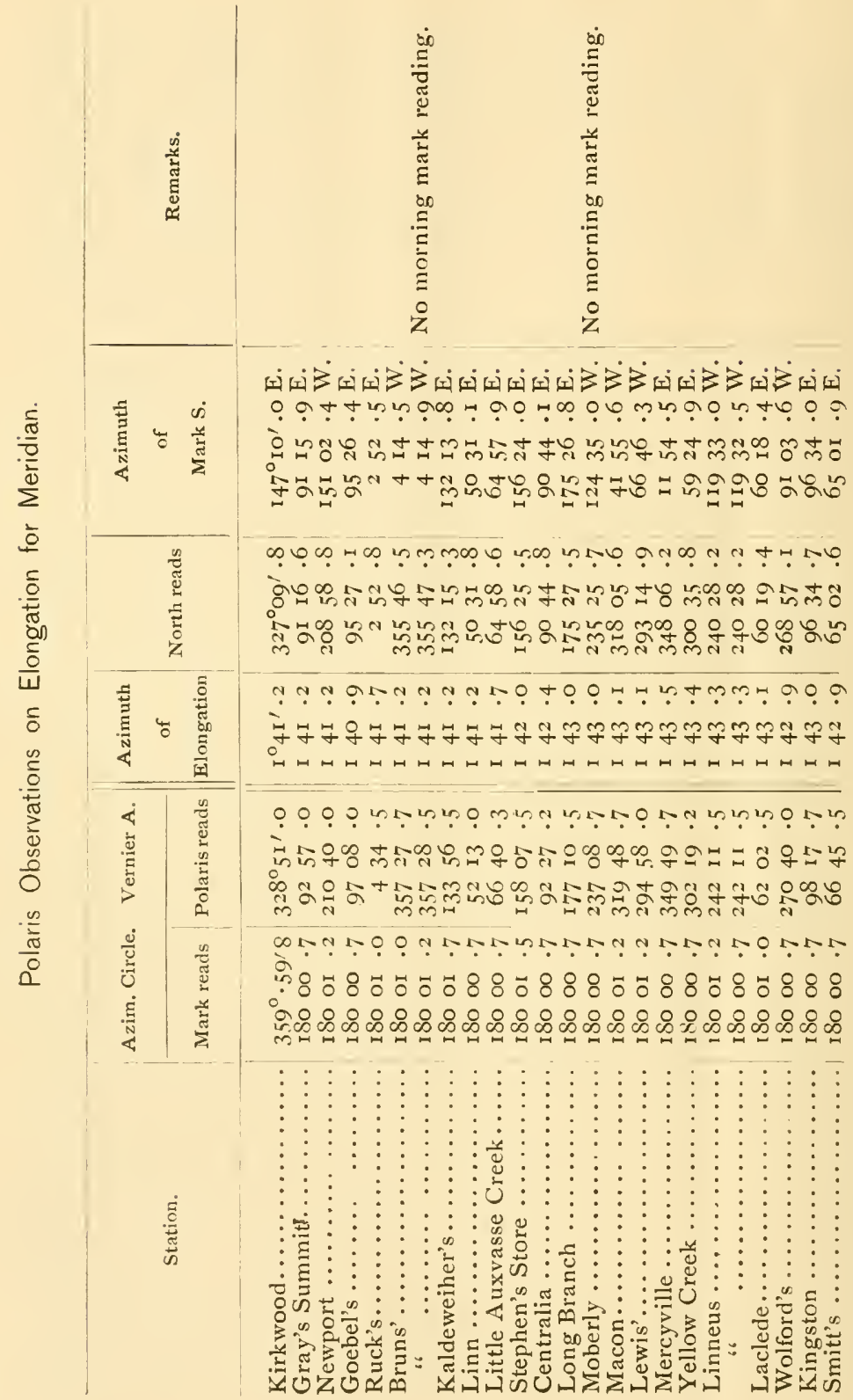




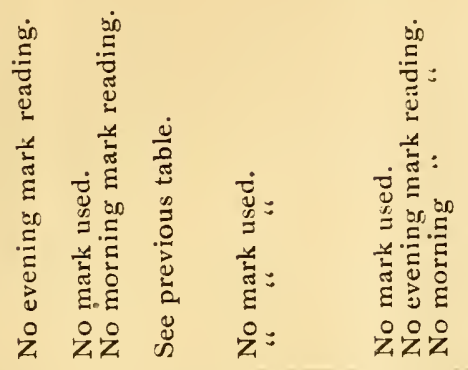

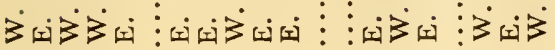

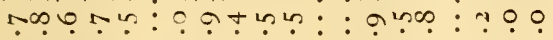

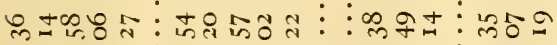

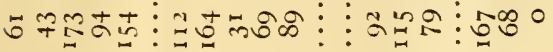

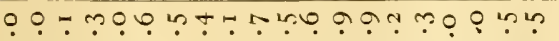

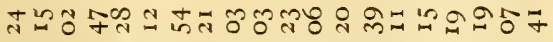

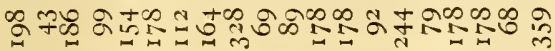

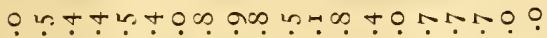

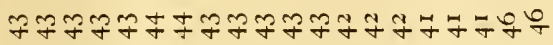

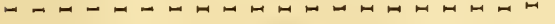

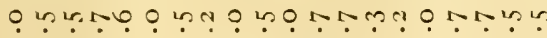

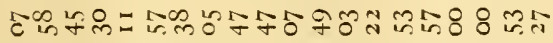

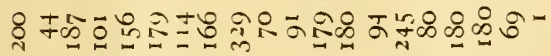

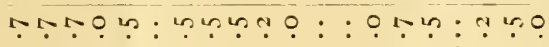
$88848 \vdots 888 \div 5 \vdots \vdots 088 \vdots 48 \pi$ ஓळ

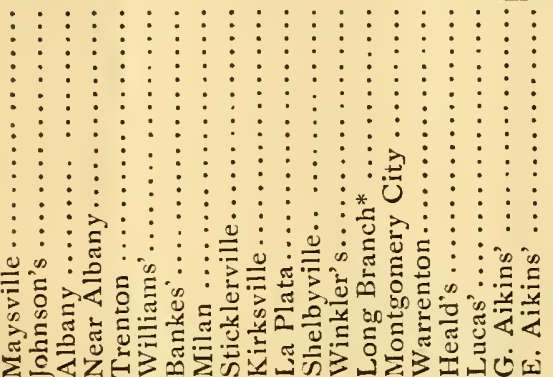




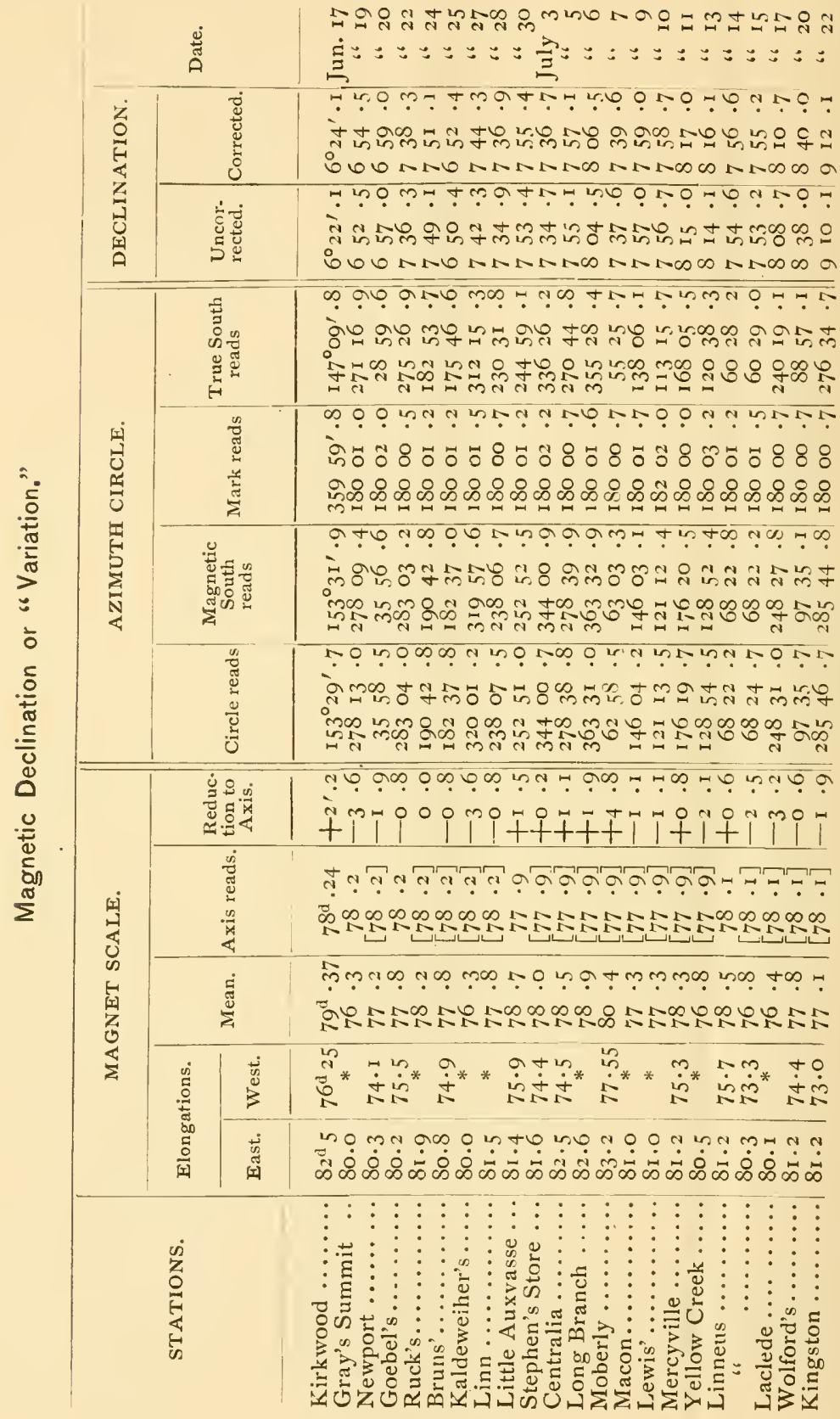




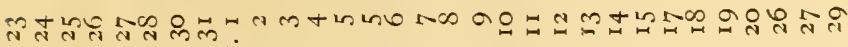

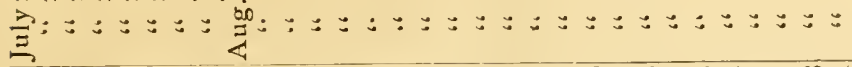

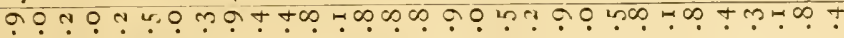

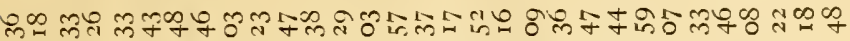

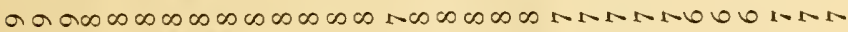

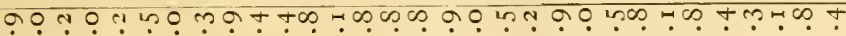

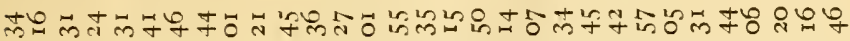

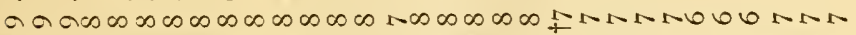

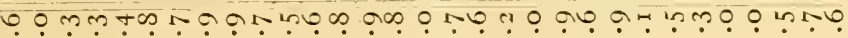

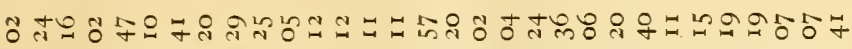
सक लु

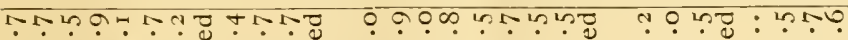

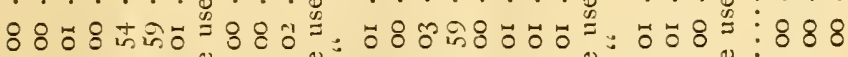
욤유

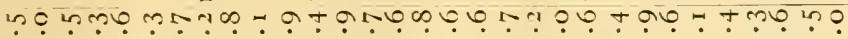

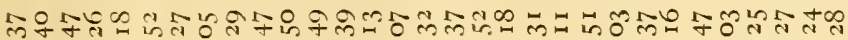

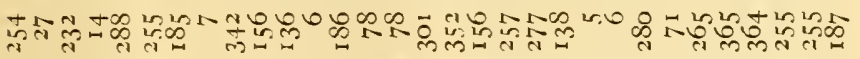

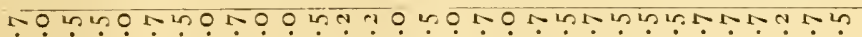

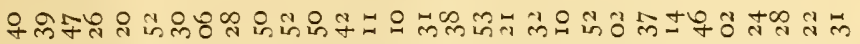
सूल

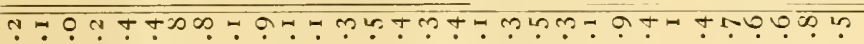

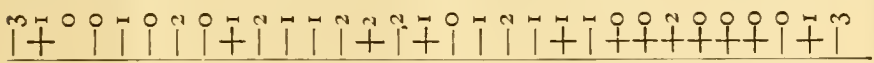

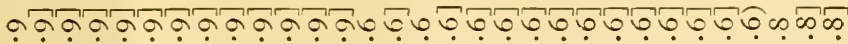

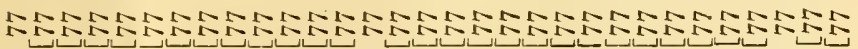

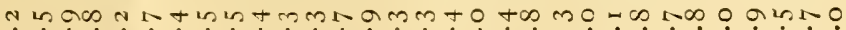

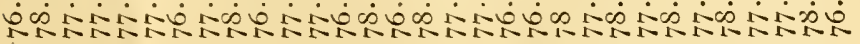

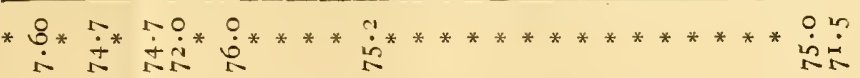

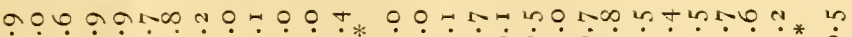

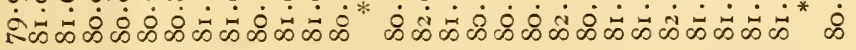

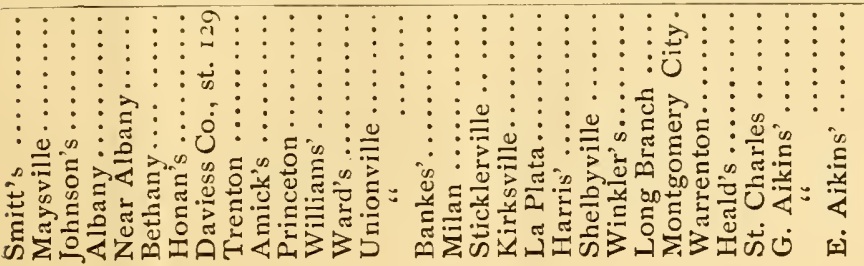




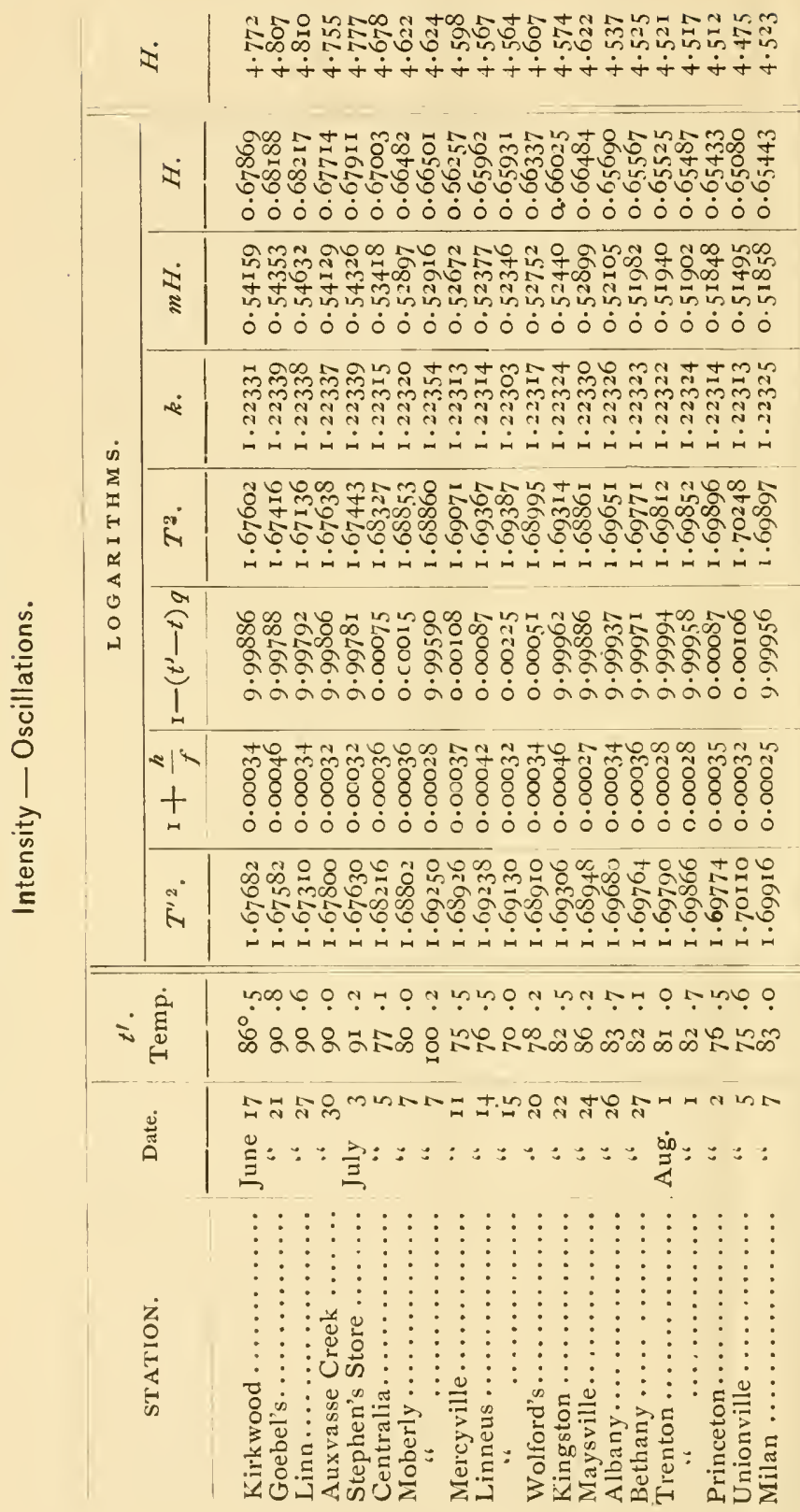




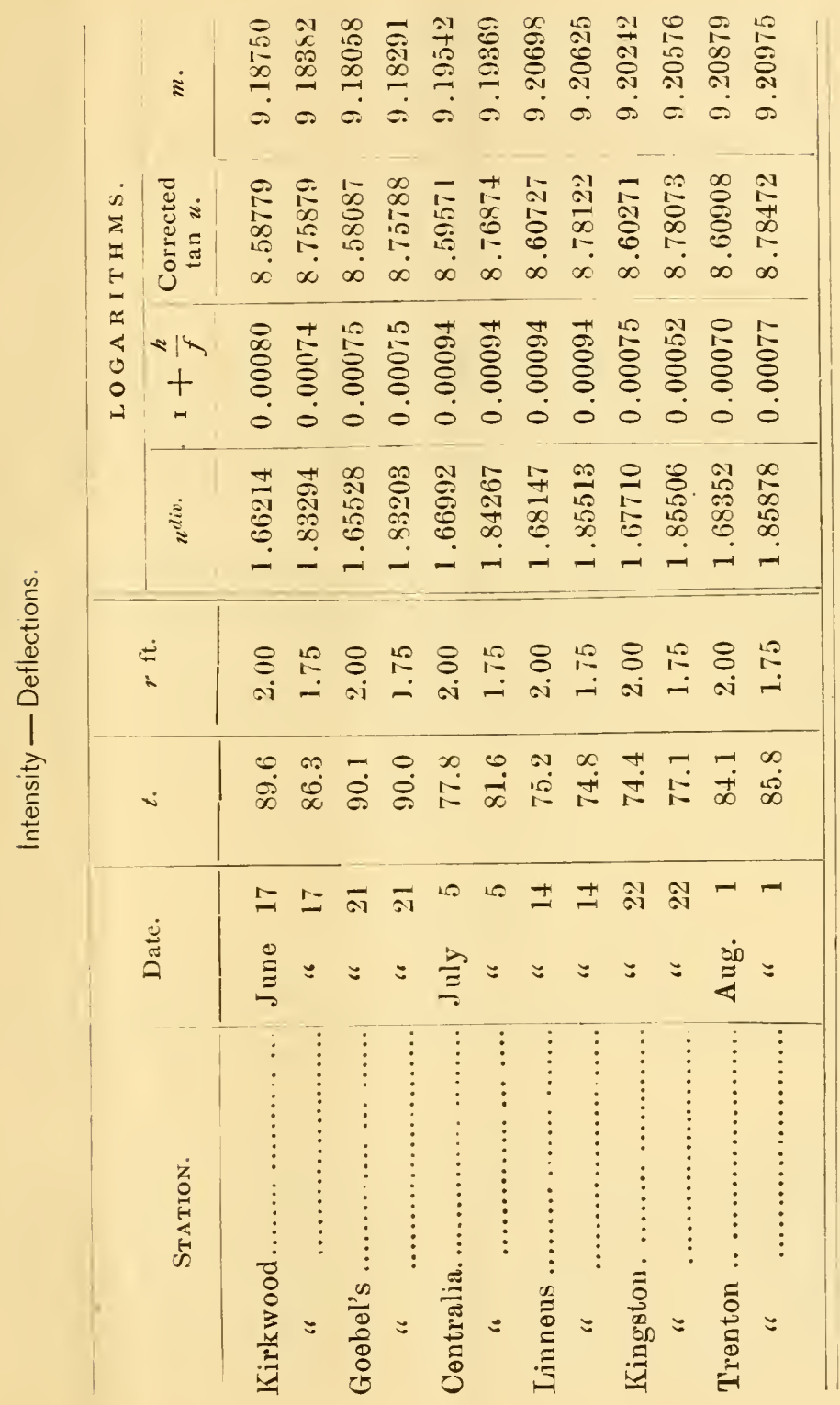


INCLINATION, OR DIP.

Needle No $:$

\begin{tabular}{|c|c|c|c|c|c|c|}
\hline \multirow{2}{*}{ STATION. } & \multicolumn{2}{|c|}{ Marked End. } & \multicolumn{2}{|c|}{ Means by Polarities. } & \multirow{2}{*}{$\begin{array}{c}\text { Resulting } \\
\text { Dip. }\end{array}$} & \multirow{2}{*}{ Date. } \\
\hline & North. & South. & Series I. & Series II. & & \\
\hline Kirkwood*.. & $68^{\circ} 591.9$ & $66^{\circ} 44, .9$ & $69^{\circ} 01, .1$ & $166^{\circ} 48, .8$ & $67^{\circ} 52, .4$ & June 17 \\
\hline Goebel's... & 6853.4 & $\begin{array}{llll}69 & 18 & .1\end{array}$ & 6858.6 & $\begin{array}{llll}69 & 12 & .8\end{array}$ & 6905.7 & \\
\hline Linn ........ & $6835 \quad .0$ & 6858.8 & $\begin{array}{lll}68 & 39 & .0\end{array}$ & $068 \quad 54 \quad .7$ & $68 \quad 46.9$ & " \\
\hline Little Aux & 6850.6 & $\begin{array}{lll}69 & 19 & .5\end{array}$ & 6854.5 & $569 \begin{array}{lll}55 & 6\end{array}$ & 6905.0 & \\
\hline Centralia... & $\begin{array}{lll}69 & 26 & .2\end{array}$ & $\begin{array}{lll}69 & 48 & .2\end{array}$ & 6944.6 & $\begin{array}{llll}669 & 29 & .6\end{array}$ & 6937.2 & July \\
\hline Moberly......... & 6525.2 & 6945.4 & $\begin{array}{llll}69 & 30 & 30\end{array}$ & 16937.8 & $69 \begin{array}{lll}35 & .3\end{array}$ & 6 \\
\hline Mercyville .............. & $\begin{array}{llll}69 & 54 & 4\end{array}$ & $\begin{array}{lll}70 & 07 & .5\end{array}$ & $\begin{array}{lll}69 & 57 & 0\end{array}$ & $\begin{array}{lllll}0 & 70 & 04 & .8\end{array}$ & $7000 \quad .9$ & " 11 \\
\hline Linneus . & $\begin{array}{llll}69 & 51 & .4\end{array}$ & 7010.0 & 6953.0 & $\begin{array}{llll}07 & 08 & .5\end{array}$ & $\begin{array}{llll}7 & 0 & 0 & .7\end{array}$ & 6 \\
\hline Wolfort's*. & 7041.2 & $\begin{array}{lll}71 & 07 & .4\end{array}$ & $70 \quad 34.9$ & $971 \quad 13.7$ & $70 \quad 54.3$ & $" 6$ \\
\hline Kingsto1 & $\begin{array}{llll}70 & 04 & .8\end{array}$ & 7015.4 & 7005.2 & 27014.8 & $\begin{array}{llll}70 & 10 & .1\end{array}$ & \\
\hline Maysville. & 6934.4 & $\begin{array}{lll}69 & 51.9\end{array}$ & 6933.8 & 86952.5 & $\begin{array}{lll}69 & 43 & .2\end{array}$ & "6 \\
\hline Albany........... & $7004 \cdot 6$ & $\begin{array}{lll}70 & 25 & .4\end{array}$ & 7008.8 & $870 \quad 21 \quad .2$ & $70 \quad 15.0$ & " 26 \\
\hline Bethany. & $\begin{array}{llll}70 & 04 & 9\end{array}$ & $70 \quad 29 \quad .0$ & $\begin{array}{llll}70 & 12 & .0\end{array}$ & $\begin{array}{llll}0 & 70 & 21 & .9\end{array}$ & $\begin{array}{llll}70 & 16 & .9\end{array}$ & " 27 \\
\hline 'Trenton.. & 7004.8 & 7024.8 & $\begin{array}{llll}70 & 04 & 04\end{array}$ & $470 \quad 25 \quad .2$ & 7014.8 & Aug. 1 \\
\hline Princeton . & $\begin{array}{llll}70 & 13 & .6\end{array}$ & 7030.0 & $70 \quad 16.2$ & $270 \begin{array}{lll}2 & 27 & .4\end{array}$ & 7021.8 & " \\
\hline Unionville.... & $\begin{array}{lll}70 & 23 & .6\end{array}$ & $\begin{array}{lll}70 & 05 & .4\end{array}$ & 7020.6 & $\begin{array}{llllll}6 & 0 & 0 & 8 & .4\end{array}$ & 7014.5 & $" 6$ \\
\hline Milan $. . . . . . \ldots \ldots \ldots \ldots . . . .$. & $\begin{array}{lll}70 & 05 & .8\end{array}$ & $\begin{array}{llll}70 & 27 & .9\end{array}$ & $\begin{array}{lll}70 & 10 & .2\end{array}$ & $270 \quad 23.4$ & $\begin{array}{lll}70 & 16 & .8\end{array}$ & 6 \\
\hline
\end{tabular}

* Some error.

Needle No. 3.

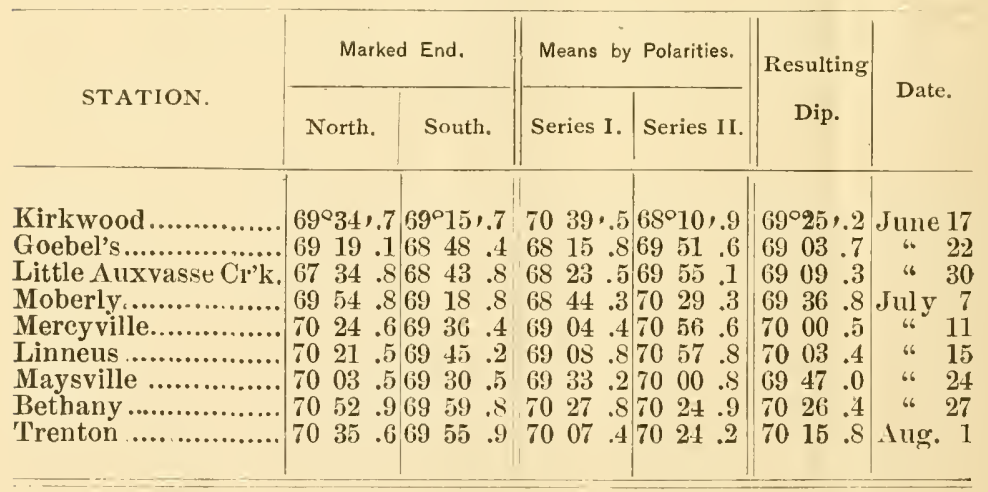




\title{
On the Expression of Electrical Resistance in Terms of a Velocity.
}

\author{
By Francis E. Nipher.*
}

If a spherical shell of radius $r$ be charged with $Q$ units of electricity, the density of electrification being $\rho$, the force $d F$ over any element $d s$ of its surface will be $2 \pi \rho^{2} d s$. This force is directed radially outward, and is due to the action of the electrification $Q$ on the quantity $\rho d s$ upon the element.

If the radius $r$ be diminished to $r^{\prime}$, the energy of the electrification will increase if $Q$ remains constant, this increase in energy being due to work done on the sphere by some external source, causing the sphere to collapse. If the element $d s$ sweeps. through a distance $d r$, the stored energy will be

$$
d E=d F d r \quad-\quad-\quad-
$$

in which both $d F$ and $d r$ are essentially negative.

Substituting in (I) the above value of $d F$ and remembering that

and

$$
\begin{aligned}
\rho & =\frac{Q}{4 \pi} r^{2} \\
d s & =r^{2} d \omega,
\end{aligned}
$$

where $d \omega$ is the solid angle subtended by the element $d s$, we have

or

$$
\begin{gathered}
d E=\frac{Q^{2}}{8 \pi} \frac{d r}{r^{2}} d \omega, \\
E^{\prime}-E=\frac{Q^{2}}{8 \pi} \iint \frac{d r}{r^{2}} d \omega,
\end{gathered}
$$

where one integration is carried over the surface of the sphere, an.? the other is carried inwards between the limits $r$ and $r^{\prime}$. Performing the integrations, we have

$$
E^{\prime}-E=\frac{Q^{2}}{2}\left(\frac{\mathrm{I}}{r^{\prime}}-\frac{\mathrm{I}}{r}\right)-\quad-
$$

But $\frac{\mathrm{I}}{2} \frac{Q^{2}}{r^{\prime}}$ is the energy of a sphere of radius $r^{\prime}$, charged with $Q$ units of electricity, and hence the potential of the sphere on itself between the limits $r$ and $r^{\prime}$ is equal to the difference in its initial and final energy.

If the sphere were connected with the ground by a wire of resistance $(R)$, the radius $(r)$ might be changed in such a manner 
as to preserve the potential $(V)$ constant. In this case a current of constant intensity would flow through the wire, and as $V=\frac{Q}{r}$ it is clear that $r$ must change at a uniform rate, or

$$
\begin{aligned}
& \frac{r-r^{\prime}}{v}=t^{\prime}-t \\
& \text { tion of the operation. } \\
& \frac{Q}{r}=\frac{4 \pi r^{2} \rho}{r}=4 \pi r \rho
\end{aligned}
$$

and

hence

$$
\rho=\frac{V}{4 \pi r} ;
$$

or

$$
d E=d F d r=2 \pi \rho^{2} d s d r=\frac{V^{2}}{8 \pi} d r d \omega,
$$

$$
E-E^{\prime}=\frac{V^{2}}{8 \pi} \iint d r d \omega=\frac{V^{2}}{2}\left(r-r^{\prime}\right) \quad-\quad \text { - }
$$

This is the stored energy during the operation. But the energy of the electrification at first was $\frac{1}{2} r V^{2}$, and at the end is $\frac{1}{2} r^{\prime} V^{2}$, so that there has nevertheless been a diminution of energy of

$$
E-E^{\prime}=\frac{V^{2}}{2}\left(r-r^{\prime}\right) \quad-\quad-\quad \text { - }
$$

It appears that, under conditions of our experiment, the sphere has less energy at the close of the experiment than at the beginning by a quantity $\frac{V^{2}}{2}\left(r-r^{\prime}\right)$, while the equal energy repre. sented by the potential of the electrification on itself was added. The total energy lost by the shell was, therefore,

$$
E=V^{2}\left(r-r^{\prime}\right)
$$

The current in the wire was, by Ohm's law,

hence

$$
C=\frac{d Q}{d t}=\frac{V}{R}
$$

$$
Q-Q^{\prime}=\frac{V}{R}\left(t^{\prime}-t\right),
$$

and hence the energy of the current during the operation was

or by $(3)$,

$$
E=\frac{V^{2}}{R}\left(t^{\prime}-t\right),
$$

$$
E=\frac{V^{2}}{R} \frac{r-r^{\prime}}{v}
$$

The expressions (6) and (7) must be equal to each other, and hence

$$
R v=\mathrm{r}, \quad \text { or } \quad R=\frac{\mathrm{I}}{\mathrm{v}},
$$

where $v$ is the constant velocity of each point in the surface of the shell during the operation. 



